\title{
Acceleration of clinician hand movements during spinal manipulative therapy
}

\author{
by \\ Geoff Gelley \\ A Thesis submitted to the Faculty of Graduate Studies of \\ The University of Manitoba \\ in partial fulfilment of the requirements of the degree of \\ MASTER OF SCIENCE
}

University of Manitoba

Faculty of Medicine

School of Medical Rehabilitation

Winnipeg

Copyright $\odot 2011$ by Geoff Gelley 


\begin{abstract}
The primary objective of this study was to determine the acceleration characteristics of spinal manipulative therapy (SMT) and whether patient anthropometric parameters correlated with the SMT kinematic parameters. For this, a wireless tri-axial accelerometer was taped to the dorsum of a clinician's hand to record the accelerations generated during clinical treatment of 95 symptomatic patients. Peak acceleration magnitudes differed significantly between spinal levels $(p<0.0001$; lumbar $>$ cervical $>$ thoracic $=$ sacroiliac). The latencies of these peaks were also significantly different $(\mathrm{p}<0.0001 ;$ lumbar $<$ cervical $=$ thoracic $<$ sacroiliac $)$. Within a given spinal level, acceleration amplitudes varied over a wide range with temporal parameters remaining relatively constant. Overall, anthropometric parameters were poorly correlated with SMT parameters. In summary, distinct acceleration amplitudes were observed across spinal levels with relatively constant temporal factors. Thus, clinicians appear to vary the magnitude rather than the duration of the SMT thrust.
\end{abstract}




\section{Acknowledgements}

The genesis of this project started with my frustration in reading the literature in regards to spinal manipulative therapy (SMT). At some point an article would refer to SMT as a high velocity and low amplitude (HVLA) manual thrust. So why the frustration? Because HVLA was anecdotal and this characteristic of SMT had not been quantified. So a few enquiries and phone calls later, I end up in the office of Dr. Dean Kriellaars. Dean with his usual enthusiasm suggests a number of approaches and ideas. But I'm still not sure. One thing leads to another and I end up in the office of Dr. Brian MacNeil. In a very short period of time I have forged a relationship with two of the brightest and keenest minds I have ever come across. Of course, it was at their urging (and also my wife's) that I decided to enroll in the MSc program in Medical Rehabilitation. For the last 5 years Dean and Brian have been my mentors and I can't thank them enough for all their help, guidance and encouragement.

A special thank you is reserved for Brian MacNeil. He undertook the task of being my advisor for this project. We met on a regular basis and no matter how busy Brian was with his teaching or his own research projects, he always made the time to make sure my questions were answered and my thesis work was on track. Through Brian's influence and attention to detail I have become a better writer and critical thinker.

A big thank you goes to Dr. Marion Alexander from the Faculty of Kinesiology. Marion graciously accepted my invitation to be on my thesis committee. Her input to my project is greatly appreciated. Two of my colleagues and friends also deserve a note of 
thanks. Drs. Ian Ledger and Alan Daien allowed me into their clinic for data collection from their patients. I am very grateful for their help and assistance.

\section{Dedication}

The last five years have been both motivating and challenging. Wearing the hats of a clinician and grad student has kept me very busy. Throughout all this, my wife Alayna and my son Reese have been my foundation. It is only through their love, support and understanding that I managed to stay focused and on purpose. Also, a big thank you goes to all my family and friends who always offered me words of encouragement. 
Table of Contents

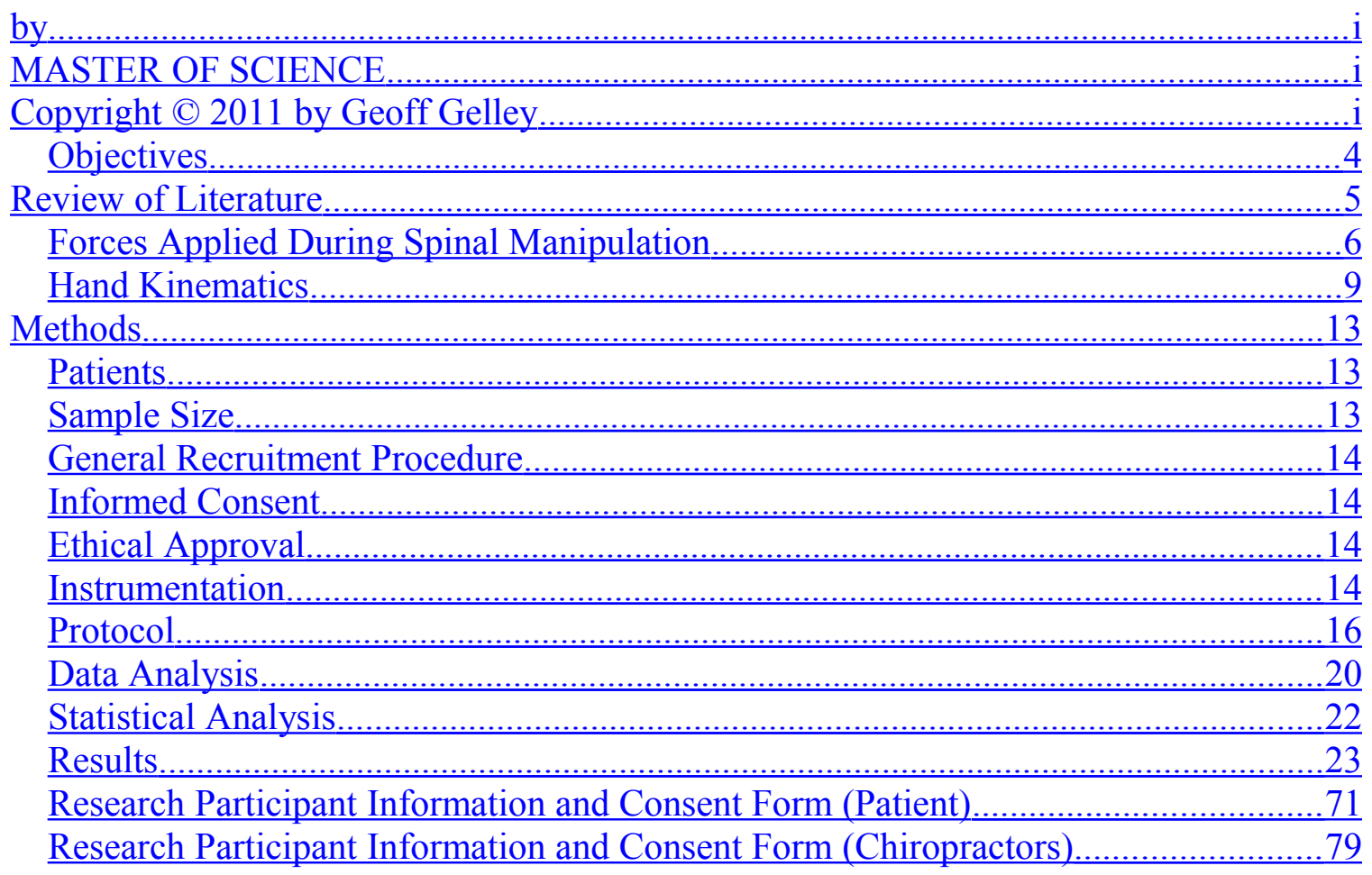

Figure 20 Acceleration and calculated velocity and displacement waveform for

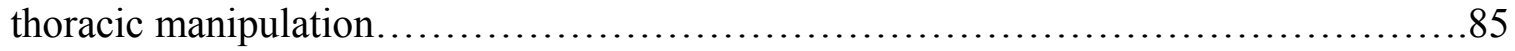

Figure 21 SMT amplitudes and latencies................................... 87

\section{List of Figures}

Figure 1 G-Link Wireless Accelerometer and Agile-Link Wireless Data Acquisition

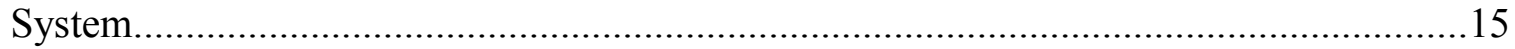

Figure 7 Average acceleration waveform for cervical manipulation trials................23

Figure 8 Average acceleration waveform for thoracic manipulation trials..............24

Figure 9 Average acceleration waveform for lumbar manipulation trials...............25

Figure 10 Average acceleration waveform for sacroiliac manipulation

trials. 
Figure 11 Peak acceleration magnitudes for each spinal

level...........................27

Figure 12 Peak acceleration latencies for each spinal level..........................29

Figure 13 Relationship between P1 peak acceleration and P1 peak latency for each spinal

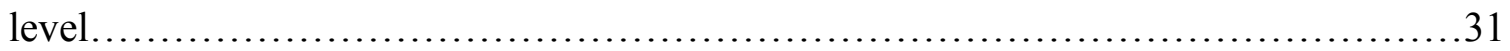

Figure 14 Correlation of P1 peak amplitude and P1 peak latency for cervical

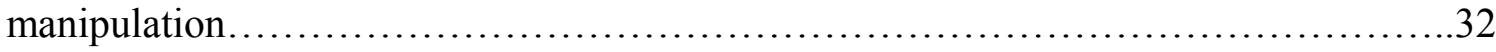

Figure 15 Correlation of P1 peak amplitude and P1 peak latency for thoracic

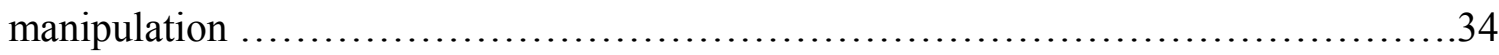

Figure 16 Correlation of P1 peak amplitude and P1 peak latency for lumbar

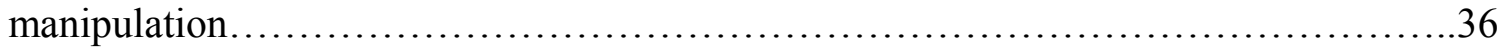

Figure 17 Correlation of P1 peak amplitude and P1 peak latency for sacroiliac

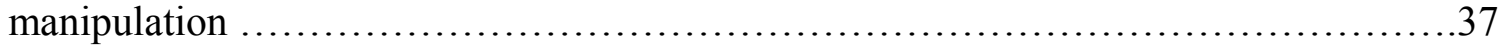

\section{List of Tables}

Table 1 Physical characteristics of subjects....................................22

Table 2 Mean velocities $\left(\mathrm{m} / \mathrm{s}^{2}\right) \pm$ standard deviation for each spinal level...............30

Table 3 Pearson correlations between P1 amplitude and P1 latency with main

components for cervical manipulation....................................... 33

Table 4 Pearson correlations between P1RS1090 and Jerk with main components for

cervical manipulation...................................................... 33 
Table 5 Pearson correlations between P1 amplitude and P1 latency with main components for thoracic manipulation.

Table 6 Pearson correlations between P1RS1090 and Jerk with main components for thoracic manipulation

Table 7 Pearson correlations between P1 amplitude and P1 latency with main components for lumbar manipulation.

Table 8 Pearson correlations between P1RS1090 and Jerk with main components for lumbar manipulation. .36

Table 9 Pearson correlations between P1 amplitude and P1 latency with main components for sacroiliac manipulation.

Table 10 Descriptive statistics of components for cervical SMT trials .38

Table 11 Pearson correlations of patient anthropometric parameters with cervical SMT main components and subcomponents

Table 12 Descriptive statistics of components for thoracic SMT trials......

Table 13 Pearson correlations of patient anthropometric parameters with thoracic SMT main components and subcomponents

Table 14 Descriptive statistics of components for lumbar SMT trials

Table 15 Pearson correlations of patient anthropometric parameters with lumbar SMT main components and subcomponents

Table 16 Descriptive statistics of components for sacroiliac SMT trials .45

Table 17 Pearson correlations of patient anthropometric parameters with sacroiliac SMT main components and subcomponents 


\section{Introduction}

Spinal manipulative therapy (SMT) is a widely administered treatment for acute and chronic conditions involving the spine and is often described as a high velocity, low amplitude (HVLA) movement (Maigne \& Guillon, 2000; Rogers \& Triano, 2003; Sran et al., 2004; Symons et al., 2002). Although most commonly associated with the chiropractic profession, an increasing number of allied health care providers such as physicians, physiotherapists, osteopaths and orthopedic specialists are also incorporating SMT into their practices (Symons et al., 2002; Tsung et al., 2005; Pickar et al., 2007).

SMT has been investigated for clinical outcomes and has been established as a clinically effective modality for the treatment of patients with low back pain, neck pain as well as cervicogenic headache and other musculoskeletal disorders (Meade et al., 1990; Shekelle et al., 1992; Koes et al., 1996; Cherkin et al., 1998; Hurwitz et al., 2002; Assendelft et al., 2003; Assendelft et al., 2004). Although research directed towards understanding the mechanisms of SMT has increased dramatically, the most fundamental biomechanical aspects of this intervention are still poorly understood (Maigne \& Guillon, 2000; Keller et al., 2003; Maigne \& Guillon, 2000; Cramer et al., 2006; Herzog et al., 1993a).

The process of SMT is typically performed by the practitioner bringing a spinal zygapophyseal joint to its end range of motion and delivering a thrust that moves the joint slightly beyond the patient's passive range of motion (the paraphysiologic space) but still within the limits of anatomic integrity. During the manipulative procedure there is often a “cracking” sound signifying successful cavitation of the joint (Herzog et al., 1993a; 
Herzog et al., 1997; Ross et al., 2004; Pickar \& Kang, 2006). Cavitation is theorized to be the result of the formation of vapor and gas bubbles within the synovial fluid upon quick separation of the joint. Although cavitation is associated with HVLA manipulation, a consensus is currently lacking as to its exact mechanism or clinical relevance (Herzog et al., 1993a).

In order to achieve the desired effect of intersegmental motion, the practitioner must prejudge the required input that will generate the intended displacement of the joint. This intent must then be translated into an appropriate hand movement generated by the clinician that is applied to a bony landmark of the target joint. The goal is to create an effective transfer process between the clinician's hand and the joint. Therefore, SMT can be viewed as having two distinct components; 1) the mechanical input delivered by the practitioner and 2) the mechanical response of the tissues to the manipulation. Similarly, the therapeutic benefit of spinal manipulation may result from the mechanical characteristics of the applied SMT input (eg. force) or the body's response to such an input (eg. vertebral displacement) (Keller et al., 2003; Kirstukas \& Backman, 1999; Keller et al., 2006b; Keller et al., 2006a; Colloca et al., 2006; Lehman et al., 2001; Triano, 2001; Solinger, 2000; Keller \& Colloca, 2002; Sung et al., 2005; Dishman et al., 2005; Pickar \& Kang, 2006; Pickar et al., 2007; Lehman \& McGill, 2001).

Of these, the effects of spinal manipulation on targeted spinal tissues has recently begun to be quantified experimentally, which is an important first step in validating the theories behind SMT (Colloca et al., 2003). Studies have examined such aspects as the spinal loads (Kawchuk et al., 1992; Herzog et al., 1993a; Kirstukas \& Backman, 1999; 
Triano, 2001; Sran et al., 2004; Tsung et al., 2005; Kawchuk et al., 2006), intradiscal pressure (Maigne \& Guillon, 2000), spinal movements and the resulting physiologic responses (Colloca et al., 2003; Colloca \& Keller, 2004; Colloca et al., 2004; Lee et al., 2005; Ianuzzi \& Khalsa, 2005b; Fernandez-de-las-Penas et al., 2007; Colloca et al., 2007).

While these studies have made considerable progress in describing the effects of SMT on the recipient tissues, comparatively little is known regarding the exact nature of the impulse delivered by the practitioner. Spinal manipulation is described as a high velocity/low amplitude thrust utilizing manual manipulative (Triano, 2001; Symons et al., 2002; Herzog et al., 2001; Kawchuk et al., 2006) and mechanically-assisted or instrument-assisted techniques (Keller \& Colloca, 2002; Keller et al., 2003; Colloca et al., 2003; Colloca et al., 2004; Colloca \& Keller, 2004; Colloca et al., 2006; Keller et al., 2006b; Keller et al., 2006a; Colloca et al., 2007). Surprisingly, the parameters of high velocity and low amplitude have never been directly measured with respect to the manipulative input.

Quantifying the kinematic aspects of SMT is essential in order to advance the study of the primary mechanisms that underlie SMT. The data from this quantification would also be useful to categorize different manipulative techniques, address concerns of patient risk and provide the basis of an evaluation tool for training future clinicians in the technique of spinal manipulation (Kirstukas \& Backman, 1999).

\section{Purpose}

The purpose of this study is to quantify acceleration of the thrusting hand during 
clinical delivery of spinal manipulative therapy. This study will provide the first such description of SMT kinematics in a clinical population. The information gained from these data will inform further investigation of the effectiveness and physiologic mechanisms of SMT.

\section{Objectives}

The primary objective of this study is to determine the acceleration characteristics of spinal manipulation at the cervical, thoracic, lumbar and sacroiliac region and whether these characteristics vary according to the level of the spine at which the manipulation is performed. The secondary objective is to determine if the anthropometric parameters of the patients receiving SMT correlate with the kinematic parameters of SMT. 


\section{Review of Literature}

It is generally agreed that the objective of delivering a spinal manipulative thrust is to cause a rapid displacement of one spinal vertebra relative to another (Haas, 1990c; Triano, 2001). In order for the mechanical input from the clinician to achieve the desired joint movement the clinician's hand must first create the intended movement. That is, the

clinician must deliver a thrust of appropriate magnitude and timing to produce the desired spinal movements. 
Forces Applied During Spinal Manipulation

Initial studies of the mechanical input from the clinician focused entirely on measuring the forces that occurred during a manipulation. The manipulative force has been described as an explicit function of the clinician's mass and impact velocity and the intrinsic stiffness and elasticity of both the clinician and patient (Haas, 1990a; Haas, 1990b). Experimentally, these forces have been measured directly or indirectly using treatment simulators (Keller \& Colloca, 2002; Sran et al., 2004; Ianuzzi \& Khalsa, 2005a; Sung et al., 2005), force platforms (Rogers \& Triano, 2003; Tsung et al., 2005) and pressure sensitive mats (Herzog et al., 1993a; Herzog et al., 1997; Kirstukas \& Backman, 1999; Herzog et al., 2001).

Several studies have examined these forces in human subjects as well as human cadavers. In cadavers, two studies have examined separately the forces at the lumbar and thoracic spinal levels. At the lumbar level, forces generated during the preload phase ranged from $61 \mathrm{~N}$ to $152 \mathrm{~N}$, whereas total forces ranged from $86 \mathrm{~N}$ to $230 \mathrm{~N}$ (Ianuzzi \& Khalsa, 2005a). Similar techniques, when applied to the thoracic spine, revealed mean pre-load forces of $82 \mathrm{~N}$ and mean peak forces of $562 \mathrm{~N}$ (Gal et al., 1995). The forces in the latter study were considerably higher than those reported for the lumbar spine and the authors speculated that the higher force magnitudes in some tissues may reflect altered tissue characteristics (reduced compliance) between cadaver studies.

More applicable to the present study, Kirstukas and Backman measured the contact pressure distribution and force magnitudes at the patient-clinician interface. Utilizing a pressure sensor interposed between the clinician and patient with the patient 
lying prone on a table outfitted with load cells, clinicians applied manipulative thrusts to the thoracic region of seven male subjects. Pre-load forces of $250 \mathrm{~N}$ to $350 \mathrm{~N}$ were measured and peak contact forces were reported as $630 \mathrm{~N}$ to $960 \mathrm{~N}$ (Kirstukas \& Backman, 1999).

Based upon the above force parameters, the peak and preload forces varied dramatically depending on which level of the spine was being manipulated. In fact, Herzog (1993a) reported that the peak forces for cervical manipulation $(118 \mathrm{~N})$ were sizably less as compared to the peak forces associated with manipulation of the thoracic spine $(399 \mathrm{~N})$ and sacroiliac joint $(328 \mathrm{~N})$. Furthermore, the treatment forces applied to patients can vary significantly between clinicians (Herzog et al., 1993a; Herzog et al., 1993b; Kawchuk et al., 1992). One laboratory has reported differing peak forces across clinician by as much as a factor of 10 (Herzog, 2000). These results suggest that the amount of force applied during spinal manipulation can vary greatly, depending not only on the treatment site manipulated but also by the variability that exists across clinicians and the technique they utilize. Notwithstanding these differences, substantially less force is generally applied to the cervical spine and then peak forces more or less increase with manipulation applied to the thoracic, lumbar and SI regions.

It is clear that considerable variation can be found in the SMT forces reported due, in part, to differences in both the regions undergoing manipulation and the technique used to deliver the manipulation. An additional source of variation arises from the use of a pressure sensing pad to measure thrust force which is the most common technique used for this purpose. These devices measure total force by integrating the individual outputs 
of the sensors that make up the sensing array. Because the contact area between the clinician's hand and the patient increases during the thrust, this will contribute to the total force measured. Unknown is what proportion of the total force is exerted directly between the key contact areas of the clinician's hand and the spinal structure targeted during the manipulation. This issue was addressed by Herzog et al (2001) by capturing the output of individual sensors within the array. Using the standard integrated output, thoracic SMT had a pre-thrust level of approximately $24 \mathrm{~N}$ and a peak force of $240 \mathrm{~N}$. However, the peak force observed from a single sensor within the primary contact point was only $5 \mathrm{~N}$. The relevance of the force exerted outside of the main contact point is unknown but is likely a major source of the variation seen across studies reporting total force. As proposed by Herzog et al (2001), much more consistent results may arise from examining local forces during SMT.

Technical issues aside, it is generally accepted that preload and peak forces during SMT vary across levels of the spinal column. Specifically, average peak and preload forces increase progressively from cervical to thoracic to sacroiliac levels (Herzog et al., 1993a; Herzog et al., 1993b). It has been suggested that a primary reason for this gradation of manipulative forces is the level of tissue resistance or stiffness encountered at each level with manipulative forces scaling accordingly. A second important observation from these studies was that, within each spinal level, both preload and peak forces were linearly related indicating that preload force is a major determinant of peak force. Preload force has been further interpreted as the means by which clinicians sense tissue resistance and thereby determine the appropriate amount of thrust needed for 
successful manipulation (Peterson \& Bergmann, 2002; Herzog et al., 1993b). Equally important from the above studies is the fact that an even greater correlation was observed between the thrust force and the peak force and, somewhat surprisingly, the preload force and thrust force were weakly correlated. Therefore, preload and thrust forces both play an important role in generating the peak forces during SMT but these appear to represent distinct, somewhat unrelated, processes. These data emphasize the need to include kinematic studies of the thrust phase to gain a more complete understanding of the SMT process.

As reviewed above, the forces associated with spinal manipulation have been evaluated reasonably well by several research groups. While the force-time histories generated from these studies are fundamental to understanding the nature of SMT, of equal importance is the study of the kinematics of how these forces are created.

\section{Hand Kinematics}

Although it may be argued that SMT kinematics can be estimated from the available force-time data using Newton's second law $(\mathrm{F}=\mathrm{ma})$ to extrapolate the acceleration component, this approach is problematic as it represents an oversimplification of the SMT process (Haas, 1990a; Herzog et al., 2001). A primary issue is determining what proportion of the clinician's body mass is actively involved in delivering the thrust. A further challenge arises from the considerable damping of the applied forces by the tissues. Given the relative low cost and ease of use of accelerometers, this technique is well suited for the study of hand kinematics during SMT. Although not yet applied to SMT input kinematics, accelerometers have been used 
to objectively measure the resultant spinal kinematics, although most of these studies are based on animal models (Gal et al., 1995; Maigne \& Guillon, 2000; Keller et al., 2003; Colloca et al., 2003; Colloca et al., 2004; Colloca et al., 2006; Keller et al., 2006b; Colloca et al., 2007; Gal et al., 1997a).

The mechanical characteristics of three commonly used chiropractic manipulation instruments (Activator IV, CAT, and Impulse) were quantified and compared in Merino sheep (Keller et al., 2006b). Accelerometers were attached to interosseous pins embedded in the spinous process of L1 and L2 of the animals. The resulting acceleration data were consistent with the simultaneously measured force-time histories. In this isolated preparation, acceleration responses were linearly related to the force settings of the various instruments. A similar relationship between the level of mechanically-delivered force and resulting acceleration was also reported by Keller and co-workers (Keller et al., 2006a). -

A similar approach has been used in human cadavers to study vertebral movements during spinal manipulation (Gal et al., 1995; Gal et al., 1997b; Gal et al., 1997a). A clinician delivered PA manipulative thrusts to T10, T11 or T12 in two unembalmed post-rigor mortis cadavers. Similar to that reported in sheep, segmental displacement increased proportionally to the input force. Interestingly, segmental acceleration has also been investigated during human surgery (Keller et al., 2003; Colloca et al., 2003; Colloca et al., 2004). Accelerometers were attached to the spinous processes of L1, L3 and L4 with interosseous pins and thrusts delivered with a manipulation instrument at varying facet joint and spinous process contact points adjacent to the 
accelerometers. Again, increased thrust forces were associated with increased acceleration at the segmental level.

In summary, the outcome of manipulative therapy to the spinal column on the recipient tissues has received considerable attention and accelerometers have provided much of the data obtained from these studies. In contrast, the input has been examined only from a force perspective which does not inform as to the kinematic nature of the hand movement that produces an SMT input. Therefore, the primary objective of this study was to use accelerometry to characterize the hand movements of clinicians during spinal manipulative therapy. 


\section{Hypotheses}

This study sought to describe the kinematic parameters of SMT by recording the acceleration of the clinician's hand during spinal manipulation at four levels of the spinal column; cervical, thoracic, lumbar and sacroiliac. Additionally, various characteristics of the SMT acceleration waveform were tested for correlations with physical attributes of the patients undergoing SMT.

A primary hypothesis of this study was that spinal manipulative therapy could be characterized by an acceleration profile that is specific to the location of the spinal region receiving the manipulation. Specifically, it was hypothesized that the magnitude of acceleration would differ between manipulation sites whereas the temporal aspects of the manipulation would not differ between sites.

A secondary hypothesis was that the acceleration profile would correspond to the anthropometric parameters of the individuals receiving the SMT. Similarly, it was expected that the magnitude of acceleration would change in proportion to the size and/or mass of the patient whereas the temporal aspects of the SMT would be relatively constant. 


\section{Methods}

\section{Patients}

Patients were included if they were over the age of 18 years and currently undergoing SMT for the treatment of mechanical neck and/or back pain. Pregnant women were excluded due to the requirement that some aspects of SMT would be performed with the client in the prone position. Patient participation beyond routine clinical treatment was limited to consent for the collection of gender, age, height, and body mass. Given the intent to describe the most representative movements that occur during SMT, this study included an experienced clinician and patients who had already undergone an initial assessment and a minimum of three treatments.

\section{Sample Size}

Sample size calculations were based on descriptive statistics from pilot data (peak amplitude mean and standard deviation; alpha $=0.05$, beta $=0.1$ ) which indicated a sample size of 7 would be adequate to detect differences between groups of subjects. However, as it was also intended to correlate SMT and anthropometric parameters, the target sample size was increased to a minimum of 20 trials for each spinal level to increase the strength of the correlation analyses. Although the initial intent was to recruit patients across several practices, recruitment at one clinic (GG) proved overwhelmingly successful $(n=95)$ while repeated attempts to recruit from additional clinics yielded very few patients $(n=4)$. As a result, a decision was made to limit data analysis to that from 
one clinic. In total, 109 SMT trials were collected with the following distribution: 37 cervical, 24 thoracic, 23 lumbar and 25 sacroiliac manipulations. The clinician was licensed with the Manitoba Chiropractors' Association with a minimum of 10 years clinical experience in spinal manipulative therapy and used manual manipulation techniques as the primary means of delivering manipulative thrusts.

\section{General Recruitment Procedure}

Initial recruitment of patients occurred through the posting of advertisements at the principal investigator's clinic. Those interested in participating in the study contacted the clinic receptionist who reviewed the details of the study and obtained informed consent.

\section{Informed Consent}

All patients reviewed and signed consent forms prior to any participation in the study. Informed consent was also obtained from the two participating clinicians. Examples of these are provided in Appendix A.

\section{Ethical Approval}

All procedures were approved by The University of Manitoba Human Research Ethics Board (Protocol Reference Number: H2008:179).

\section{Instrumentation}

A wireless tri-axial accelerometer was used to record acceleration data during SMT. The device consisted of a G-Link Wireless Triaxial Accelerometer and the Agile- 
Link Wireless Data Acquisition System (Microstrain Inc., Williston, VT. USA; Fig.1). The accelerometer streamed data in real time from the three active channels with a sampling rate of $617 \mathrm{~Hz}$ per channel. The data was received by the Agile-Link Wireless Data Acquisition System which is powered through a laptop computer USB port and analyzed with Agile-Link software. Each channel represents one of three coordinates of the $\mathrm{x}, \mathrm{y}$ and $\mathrm{z}$ orthogonal model.

Figure 1 G-Link Wireless Accelerometer and
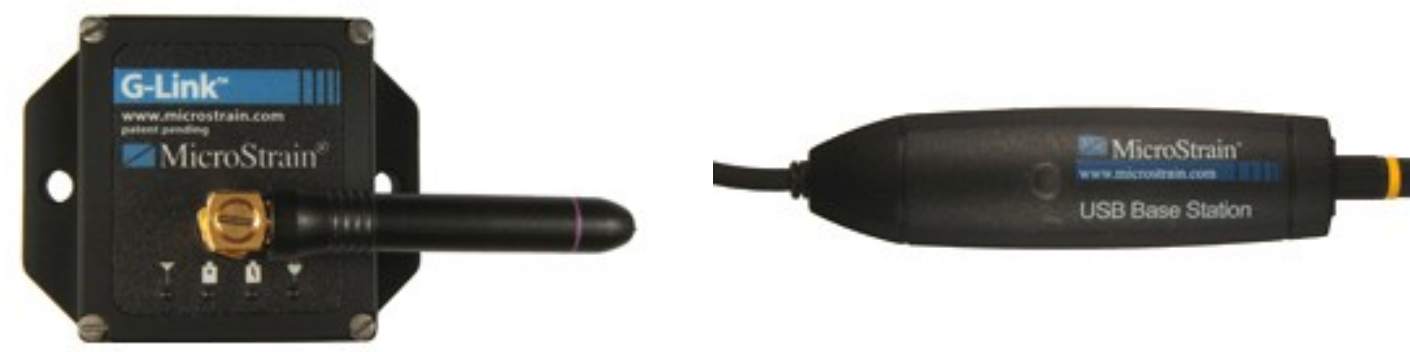

Agile-Link Wireless Data Acquisition System

The wireless accelerometer was attached to the dorsal aspect of the clinician's hand in alignment with the long axis of the third metacarpal using adhesive tape (Fig. 2). This position was chosen for several reasons including the ability to align the accelerometer with a distinct anatomical feature of the clinician's hand and the relative thinness of the underlying tissue layer. However, the primary reason for this orientation was that it ensured one of the axes of the accelerometer was aligned with the primary direction of movement during each of the SMT techniques. The specific axis which best captured the different SMT techniques were confirmed during pilot studies using high speed video recordings. 


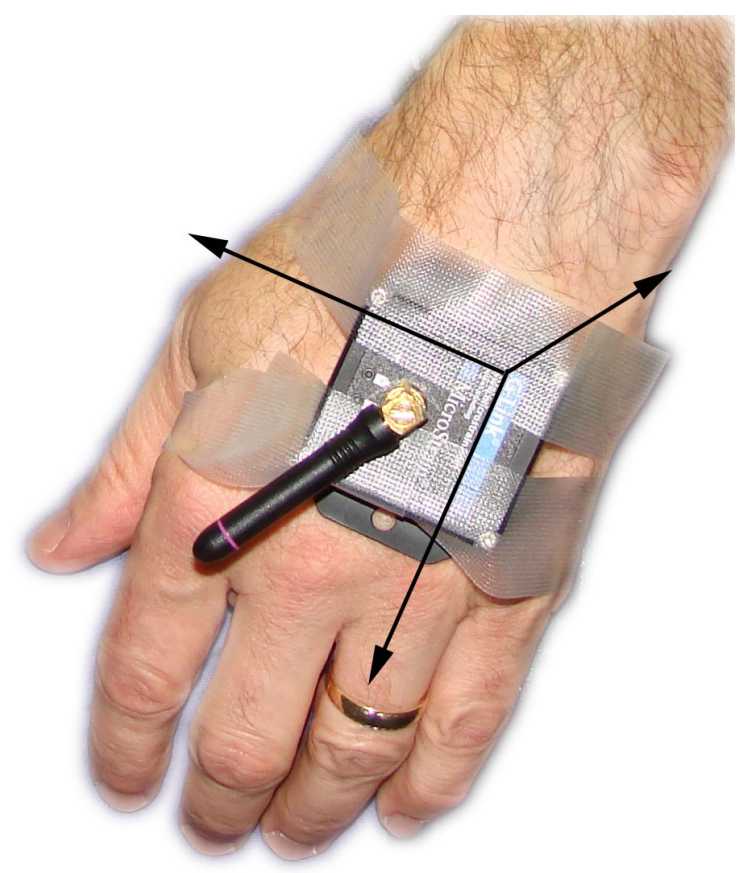

Figure 2. Accelerometer with 3 channel orientations

Calibration of the G-Link accelerometer was performed at the beginning of each experimental session utilizing the gravitational orientation method (Webber \& Kriellaars, 2004). The accelerometer was positioned to create $\mathrm{a}+1 \mathrm{~g}$ or $-1 \mathrm{~g}$ signal for each of the three axes. The signal for each of these reference positions was used to convert the accelerometer signals to $+/-9.8 \mathrm{~m} / \mathrm{s}^{2}$. The midpoint of these values was used to indicate the accelerometer signal at zero "g" and this value was subtracted from all accelerometer values to remove any offset in the resulting waveform that occurred when converting the raw acceleration to "g".

\section{Protocol}

After obtaining informed consent, anthropometric parameters were collected from all patients by clinic staff other than the treating clinician. Patients then proceeded to their 
usual appointment with the clinician. As per a typical visit, the clinician completed an initial re-assessment including the patient's subjective information and any objective findings (ROM, palpatory findings, etc.) along with confirmation of the spinal segment(s) to be manipulated. Once the spinal segment(s) were identified and the manipulative procedure chosen, the patient was placed in the required position on a chiropractic treatment table. The accelerometer was then attached the clinician's hand with adhesive tape and an assistant initiated the data collection process on a laptop computer. The clinician then delivered the preload with a brief pause prior to the SMT thrust. Acceleration data were reviewed immediately to verify a successful collection. This process was repeated if a second or third spinal segment was to be manipulated. Presence or absence of an audible sound was recorded with each manipulation along with the spinal segment manipulated.

\section{Manipulative Procedures Evaluated}

Cervical (rotary break): The patient was supine with the cervical spine in the neutral position. The index finger of the clinician made contact over the posterolateral aspect of the zygapophyseal joint selected. The clinician then cradled the patient's head with the other hand. Gentle ipsilateral side-flexion and contralateral rotation to the targeted side was introduced until slight resistance to further rotation perceived. A rotational thrust was then directed medially in the direction of the participant's contralateral shoulder (Figure 3). 


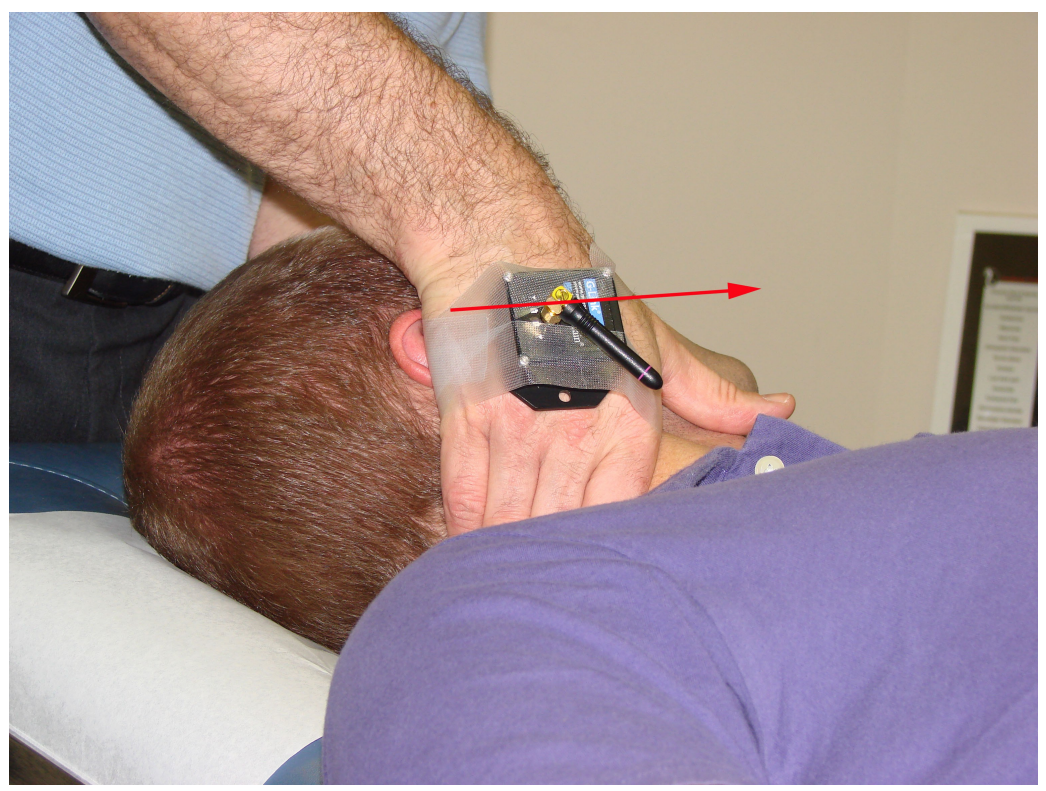

Figure 3. Cervical manipulation with thrust vector

Thoracic (bilateral thenar): With the patient in the prone postion the clinician placed the thenar eminences of both hands to make contact over the transverse processes of the target vertebra. The thrust was applied with both hands in a posterior-anterior direction (Figure 4).

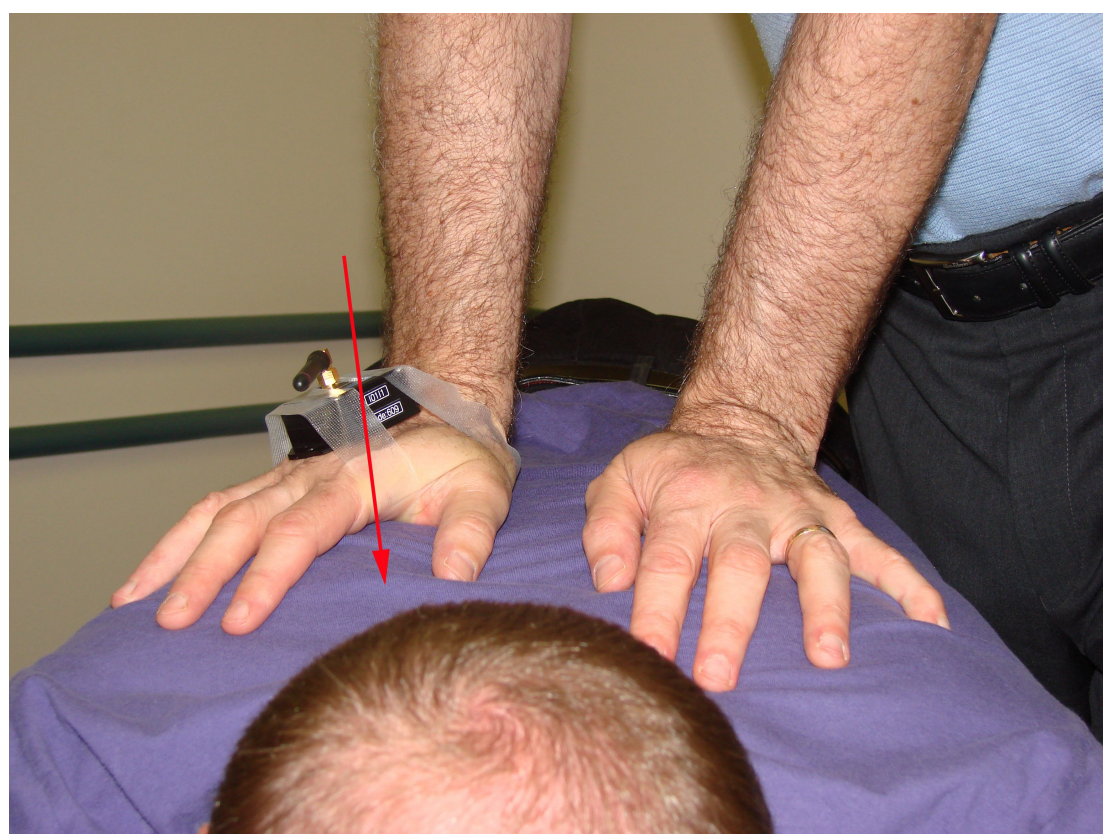


Figure 4. Thoracic manipulation with thrust vector

Lumbar (spinous pull): The patient was placed in the lateral recumbent position. The clinician made contact with the middle finger over the target vertebral spinous process on the side closest to the table and the clinicians opposite hand was placed over the participant's superior shoulder to stabilize. The thrust was generated using a body drop directed through the longitudinal axis of a forearm which is the same axis as the accelerometer placement on the clinician's hand (Figure 5).

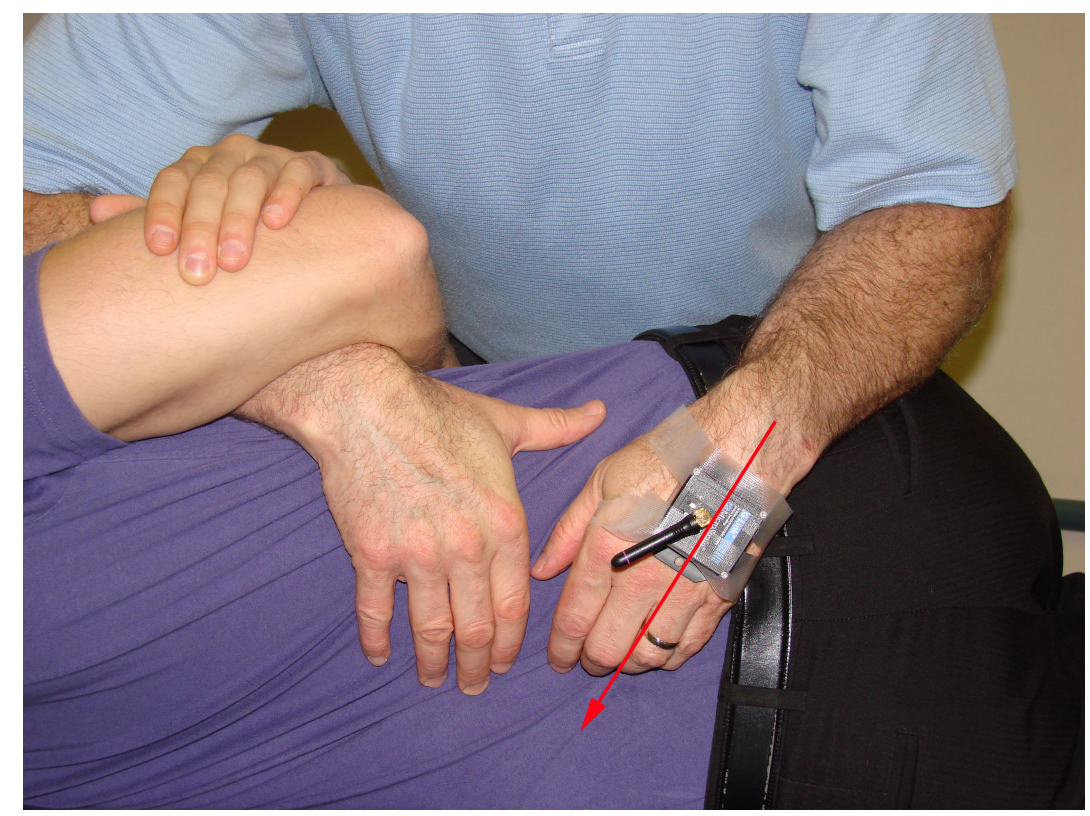

Figure 5. Lumbar manipulation with thrust vector

Sacroiliac: The clinician's non-contact hand stabilized the patient's superior shoulder in side-lying. The clinician made a pisiform contact over the PSIS of the involved SI joint. The thrust was produced by the contact hand in an anterior direction while quickly dropping the body onto the pelvis and the line of drive was down the 
longitudinal axis of the participant's flexed thigh (Figure 6).

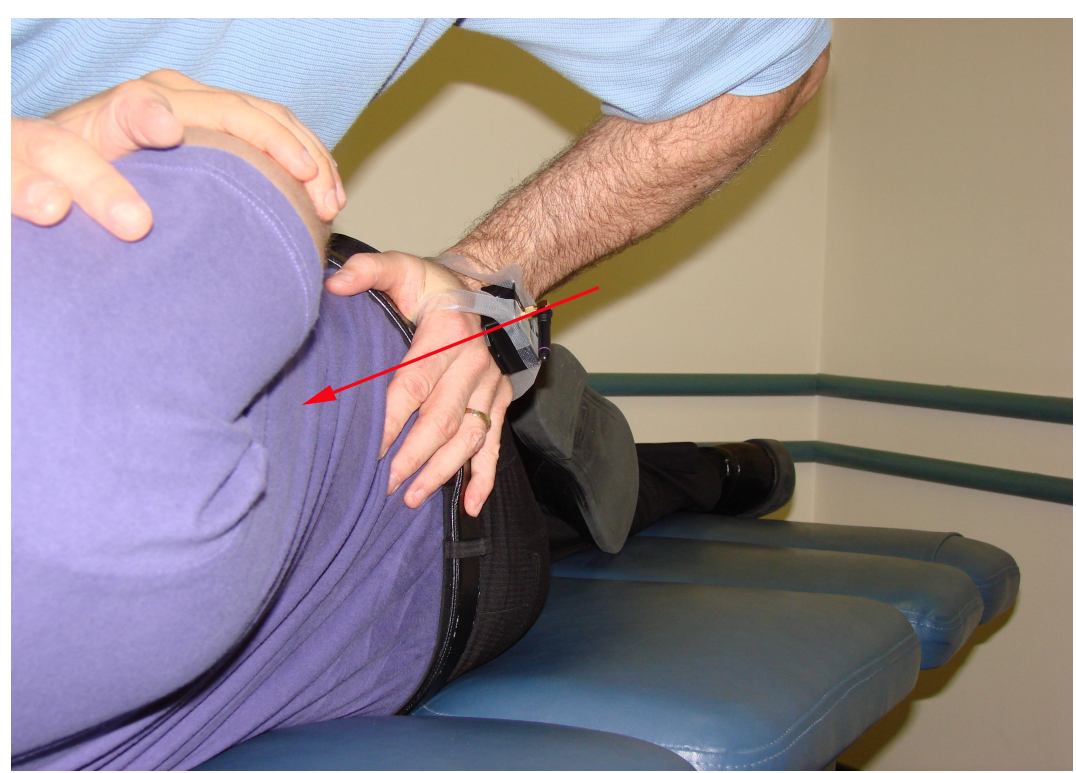

Figure 6. Sacroiliac manipulation with thrust vector

\section{Data Analysis}

Raw acceleration data was imported into Microsoft Excel and plotted. A one second epoch that contains the SMT event was isolated from the data file and exported to DADiSP/SE 6.5 (Newton, MA) for bandpass filtering $(0.1-50 \mathrm{~Hz}$, 4th order Butterworth). Filtered data was then returned to Excel. Further analysis was completed only on the channel which matched the axis of the SMT thrust. While it is recognized that the resulting movements of the underlying joint are not restricted to a single axis, the thrust from the clinician is applied in a linear manner and the positioning of the accelerometer placed one of its axes in line with the direction of the SMT thrust. Indeed, the likely outcome at the tissue level is a combination of linear and angular displacements (Haas, 1990c; Herzog, 2000; Kawchuk \& Perle, 2009), however, the intent of the present study 
was to characterize the input from the clinician and not the response of the tissues to that input.

The onset of the SMT event was defined relative to the acceleration-derived jerk waveform. For this, jerk was calculated from the acceleration data through numerical differentiation. A baseline segment of the jerk data was then used to calculate the standard deviation of the jerk baseline. A threshold equivalent to two standard deviations was then generated and the onset in the acceleration data was taken as the point at which the jerk waveform exceeded the 2 standard deviation threshold. The typical waveform consisted of a triphasic response from which various parameters were derived (Figure 1, Appendix B) including the peak acceleration amplitudes of P1, P2 and P1P2 as well as their associated latencies. Additional primary variables were P1 duration and P1 area under the curve (AUC). Further characterization of the SMT waveforms included the slope of the rising segment of the P1 phase. Specifically, the slope of the rising phase of P1 was calculated between $10 \%$ and $90 \%$ of the peak amplitude (P1RS1090). These components of the P1 phase were used to capture the initial part of the manipulative event to examine if the P1 phase (clinician input) was coupled to the P2 phase (tissue response). The peak of the jerk waveform during the rising phase of P1 was also analyzed. The third phase of the acceleration waveform, P3, was not included in the analysis as this corresponds to the resolution phase following the treatment thrust and is not considered clinically important (Herzog, 2000).

Peak velocity and displacement were also estimated using numerical integration. Peak velocity was calculated at all spinal levels while the displacement analysis was 
restricted to the thoracic manipulations since the SMT technique used at the thoracic level permitted the delivery of a posterior-to-anterior thrust that, for the most part, started and finished with the hand in the same position and orientation. Without this feature, the estimates of displacement obtained through double numerical integration of the accelerometer waveform contain excessive error. A detailed description of preliminary steps undertaken to determine the analysis parameters is included in Appendix B.

\section{Statistical Analysis}

Statistical analysis was performed using Statistica software (StatSoft, Tulsa OK). Mean values for acceleration parameters were compared between spinal levels using a one-way analysis of variance (ANOVA) with Tukey's post-hoc tests to determine significant pair-wise comparisons. Pearson correlations were performed to determine whether the SMT kinematics were associated with anthropometric characteristics of the patients undergoing SMT. Statistical significance was defined as an alpha level of $p<0.05$. 


\section{Results}

The study sample ( $\mathrm{n}=95$ patients) was drawn from the principal investigator'schiropractic clinic. All patients were symptomatic and had already undergone an assessment and a minimum of three treatments for neck and/or back pain. Patient physical characteristics can be seen in Table 1.

Table 1. Physical characteristics of subjects (mean \pm standard deviation).

\begin{tabular}{|c|c|c|c|c|}
\hline & Age (yr) & Height (m) & Weight (kg) & BMI (kg/m2) \\
\hline $\begin{array}{l}\text { Male } \\
(n=25)\end{array}$ & $48.22 \pm 13.44$ & $1.77 \pm .06$ & $91.33 \pm 13.00$ & $29.01 \pm 3.29$ \\
\hline $\begin{array}{l}\text { Female } \\
(n=70)\end{array}$ & $48.47 \pm 10.30$ & $1.63 \pm .08$ & $77.69 \pm 17.32$ & $29.23 \pm 5.56$ \\
\hline
\end{tabular}

The acceleration-time histories displayed a similar tri-phasic pattern across all spinal levels. The only exception to this generalized pattern was that for SMT at the SI level where a bi-phasic P1 segment was often seen. Specific details of the characteristic waveforms for each spinal level are provided below. 


\section{Cervical SMT trials}

A total of 37 cervical trials were collected from 21 patients as several patients received treatment at more than one cervical spinal level. The mean peak amplitude of the P1 component of cervical SMT was $8.89 \pm 3.15 \mathrm{~m} / \mathrm{s}^{2}$ with a latency of $58 \pm 13 \mathrm{~ms}$ (Figure 7). The mean peak amplitude of the $\mathrm{P} 2$ phase was $-8.74 \pm 3.00 \mathrm{~m} / \mathrm{s}^{2}$ with a latency of $132 \pm 29 \mathrm{~ms}$. The peak velocity for cervical manipulation was $0.41 \pm 0.14 \mathrm{~m} / \mathrm{s}$ with a latency of $87 \pm 20 \mathrm{~ms}$.

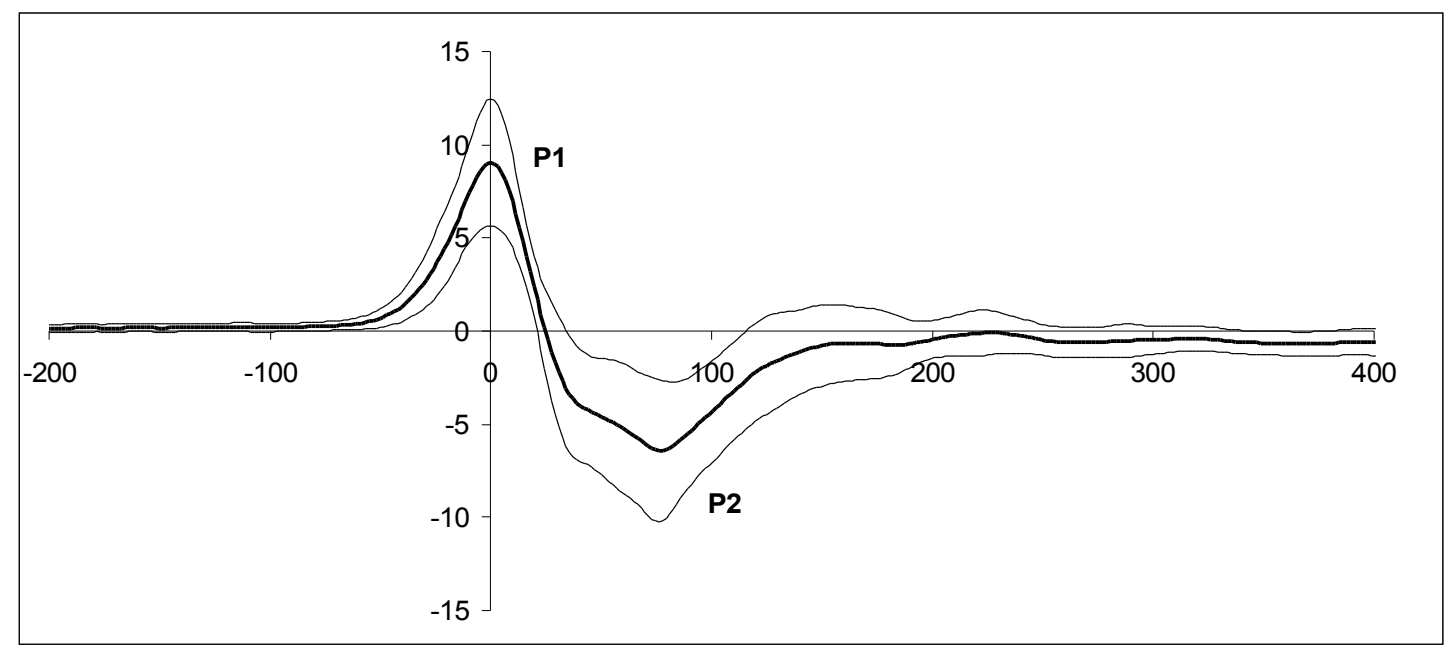

Figure 7. Average acceleration waveform for cervical manipulation trials. Individual waveforms were assigned a time value of $0 \mathrm{~ms}$ at $\mathrm{P} 1$ peak and the corresponding values were averaged for $200 \mathrm{~ms}$ prior to $\mathrm{P} 1$ peak and $400 \mathrm{~ms}$ following P1 peak. Acceleration is plotted in $\mathrm{m} / \mathrm{s}^{2}$ for mean (heavy line) and \pm 1 standard deviation (light lines).

\section{Thoracic SMT Trials}

A total of 24 thoracic trials were collected from 24 patients. The mean amplitude of the P1 component of thoracic SMT was $2.80 \pm 1.01 \mathrm{~m} / \mathrm{s}^{2}$ with a latency of $71 \pm 15 \mathrm{~ms}$ (Figure 8). The mean amplitude of the P2 phase of thoracic SMT was $-4.09 \pm .98 \mathrm{~m} / \mathrm{s}^{2}$ with a latency of $147 \pm 17 \mathrm{~ms}$. The mean peak velocity for thoracic manipulation was 
$0.18 \pm 0.04 \mathrm{~m} / \mathrm{s}$ with a latency of $104 \pm 12.5 \mathrm{~ms}$. The mean peak displacement was 12 $\pm 3.02 \mathrm{~mm}$ with a latency $158 \pm 15.4 \mathrm{~ms}$.

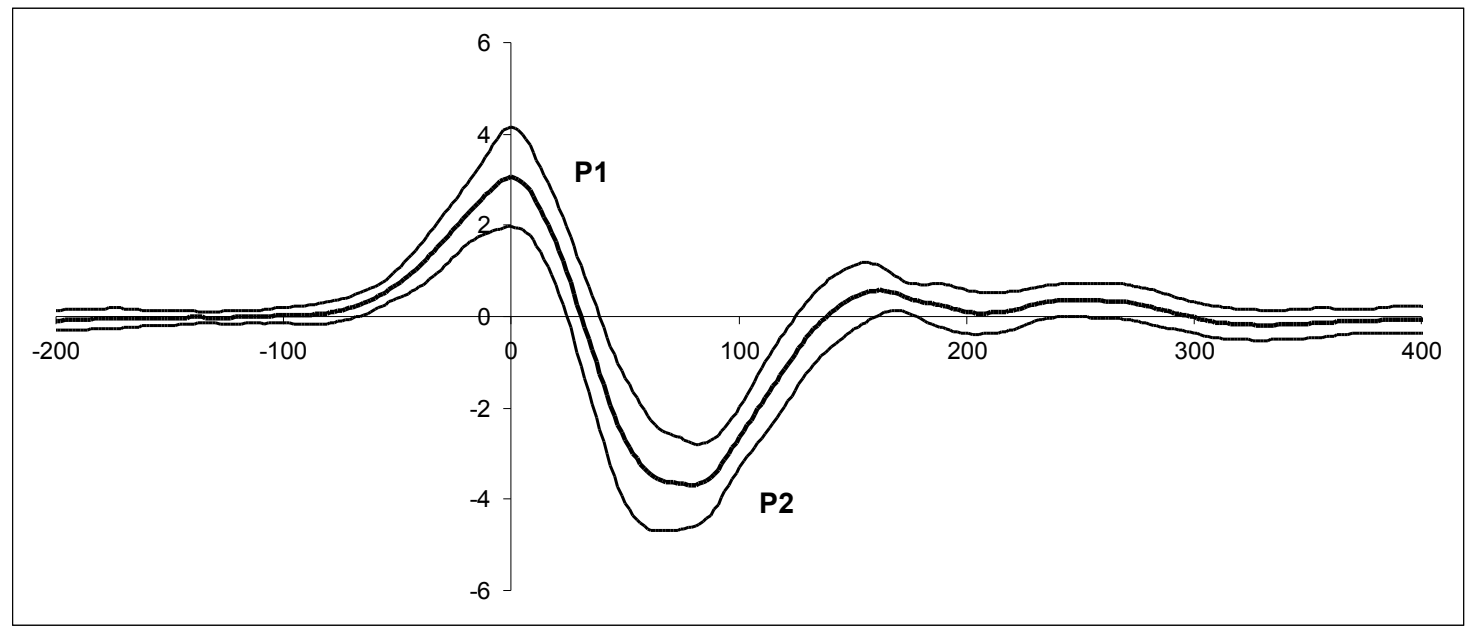

Figure 8. Average ( \pm 1 standard deviation) acceleration waveform $\left(\mathrm{m} / \mathrm{s}^{2}\right)$ for thoracic manipulation trials plotted as described in Figure 1.

\section{Lumbar SMT Trials}

A total of 23 lumbar trials were collected from 23 patients. The mean amplitude of the P1 component of lumbar SMT was $17.87 \pm 7.06 \mathrm{~m} / \mathrm{s}^{2}$ with a latency of $49 \pm 13 \mathrm{~ms}$ (Figure 9). The mean amplitude of the P2 peak of SMT at the lumbar spinal level was $-11.67 \pm 5.75 \mathrm{~m} / \mathrm{s}^{2}$ with a latency of $92 \pm 19 \mathrm{~ms}$. The mean peak velocity for lumbar manipulation was $.440 \pm .97 \mathrm{~m} / \mathrm{s}$ with a latency of $114 \pm 11 \mathrm{~ms}$. 


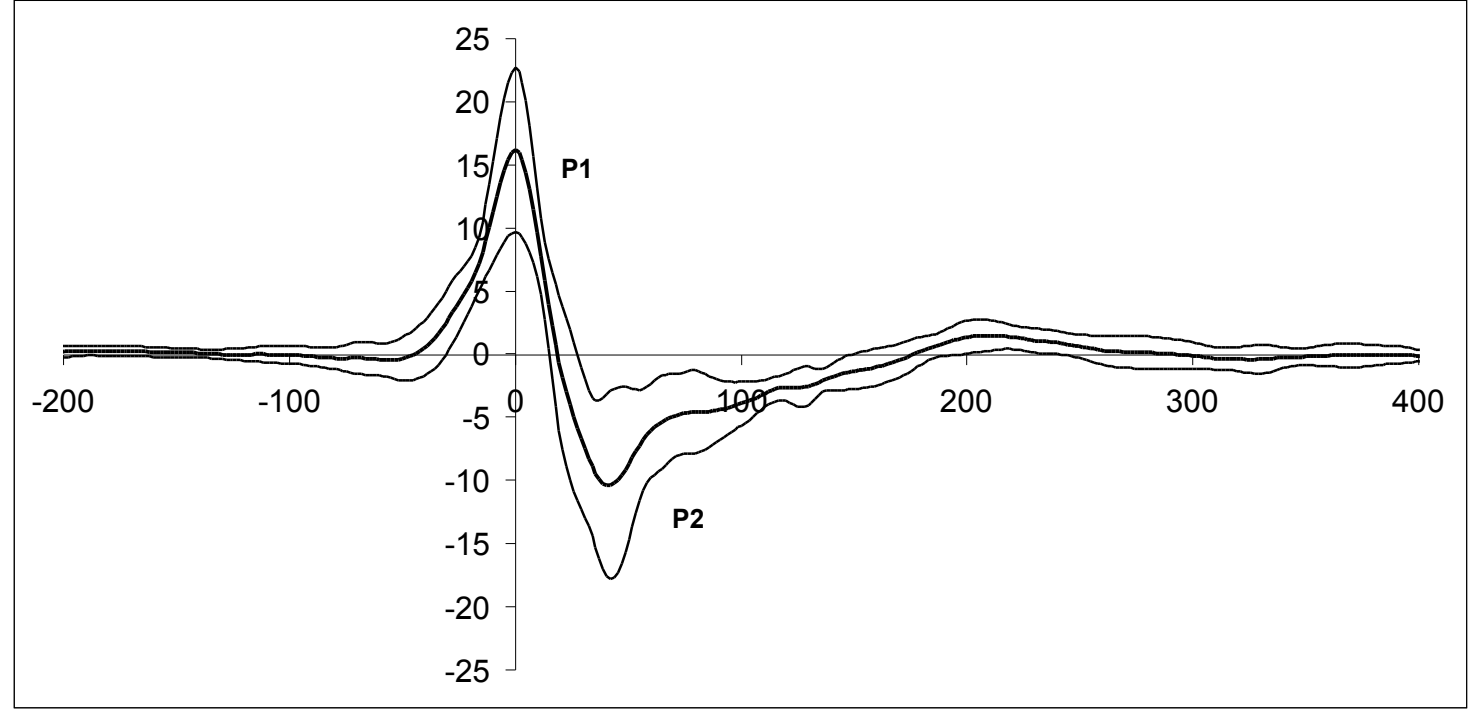

Figure 9. Average ( \pm 1 standard deviation) acceleration waveform $\left(\mathrm{m} / \mathrm{s}^{2}\right)$ for lumbar manipulation trials plotted as described in Figure 1.

\section{Sacroiliac SMT Trials}

A total of 25 sacroiliac trials were collected from 25 patients. The mean amplitude of the P1 component of sacroiliac SMT was $4.38 \pm 2.40 \mathrm{~m} / \mathrm{s}^{2}$ with a latency of $132 \pm 37$ ms (Figure 10). The mean peak amplitude of the P2 component of sacroiliac SMT was $-4.68 \pm 1.64 \mathrm{~m} / \mathrm{s}^{2}$ with a latency of $205 \pm 38 \mathrm{~ms}$. The mean peak velocity for sacroiliac manipulation was $.189 \pm .97 \mathrm{~m} / \mathrm{s}$ with a latency of $150 \pm 30 \mathrm{~ms}$. Notable within the SI manipulations was a dual peak within the P1 phase of the thrust. High speed video recordings confirmed that this resulted from a lateral off-axis motion in the middle of the rising portion of the $\mathrm{P} 1$ phase. Presumably, the relatively flat contact area between the clinician's hand and the SI region, combined with limited ability of the shoulder of the manipulating arm to both generate the thrust and stabilize the joint, permits lateral displacement of the hand during the thrust phase. 


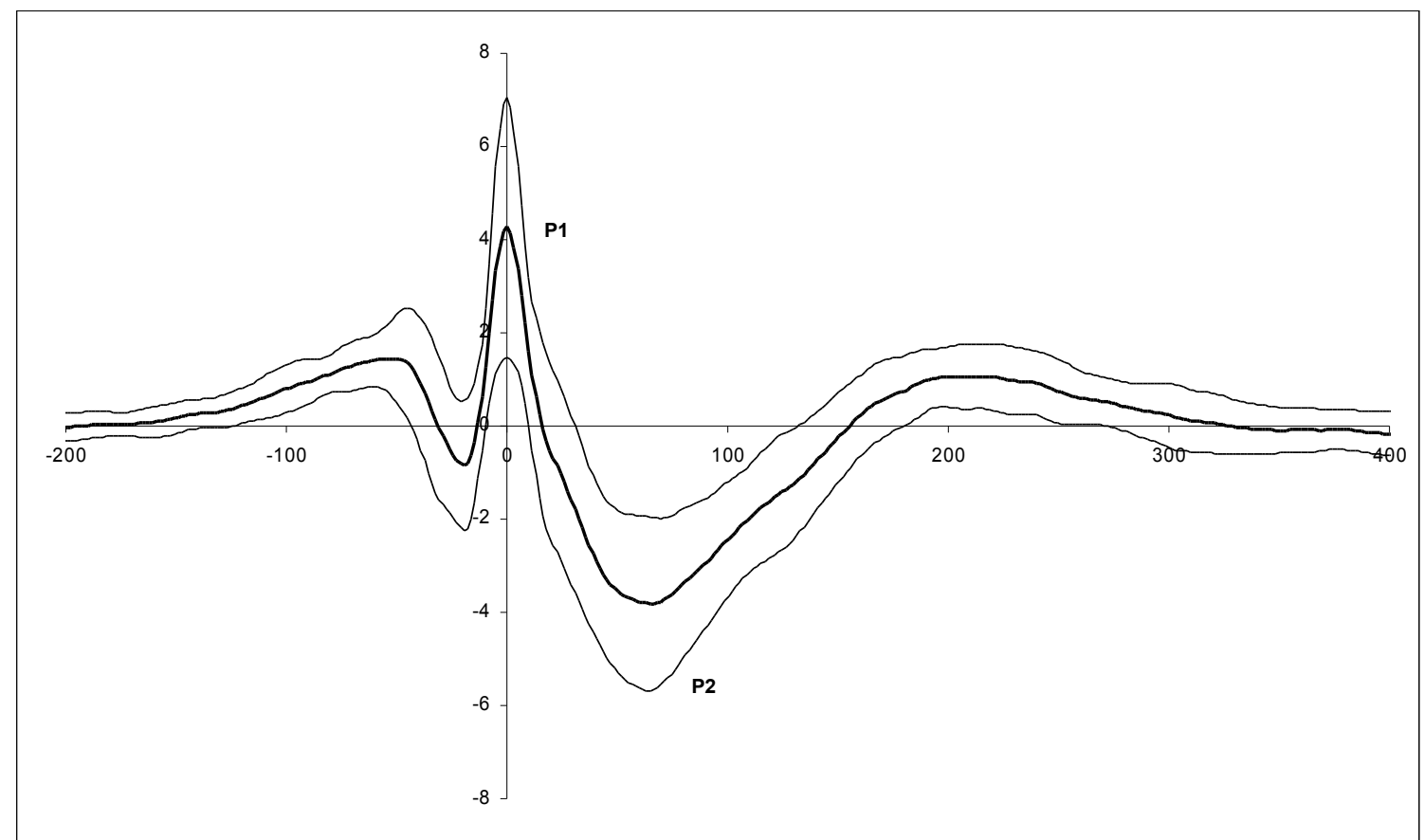

Figure 10. Average ( \pm 1 standard deviation) acceleration waveform $\left(\mathrm{m} / \mathrm{s}^{2}\right)$ for sacroiliac manipulation trials plotted as described in Figure 1.

\section{Characteristics of SMT at Different Spinal Levels}

A primary objective of the present study was to compare SMT characteristics across different levels of the spinal column. For this, one-way ANOVAs were completed for each of the SMT parameters derived from the acceleration waveforms with Tukey's post-hoc tests used to determine significant pair-wise comparisons.

A strong effect of spinal level was present for the parameters which described the amplitude of hand acceleration during SMT; P1 peak amplitude $\left(\mathrm{F}_{3,105}=68.83\right.$, $\mathrm{p}<0.0001)$, P2 peak amplitude $\left(\mathrm{F}_{3,105}=28.76, \mathrm{p}<0.0001\right)$ and $\mathrm{P} 1-\mathrm{P} 2$ peak to peak amplitude $\left(\mathrm{F}_{3,105}=62.45, \mathrm{p}<0.0001\right)$. Post-hoc analysis revealed an identical pattern across all of these amplitude characteristics (Figure 11). Specifically, similar magnitudes were seen 
between thoracic and sacroiliac manipulations $(\mathrm{p}>0.5)$. Cervical manipulations consistently produced acceleration amplitudes that were greater than those seen at either the thoracic or sacroiliac levels $(\mathrm{p}<0.001)$ and the amplitudes occurring during lumbar manipulations were greater than all other levels $(\mathrm{p}<0.01)$. A related outcome, P1 AUC, also differed significantly across spinal levels $\left(\mathrm{F}_{3,105}=70.71, \mathrm{p}<0.0001\right)$ and shared the same pattern as above regarding pair-wise differences between the anatomical locations. Additional analysis was completed for each spinal level to determine whether gender differences were present for the magnitude-related parameters of acceleration. There were no significant differences between gender and the amplitude-based acceleration parameters for the cervical and thoracic levels $(\mathrm{p}>0.05)$. Significant gender differences were observed such that males had larger magnitude-related parameters at the lumbar [P1 amp (p<0.002); P1P2 Amp ( $p<0.004) ;$ P1AUC $(p<0.001)]$ and SI [P1 amp $(p=0.014)$; P1P2 Amp $(\mathrm{p}<0.040)$; P1AUC $(\mathrm{p}<0.01)]$ levels during SMT.

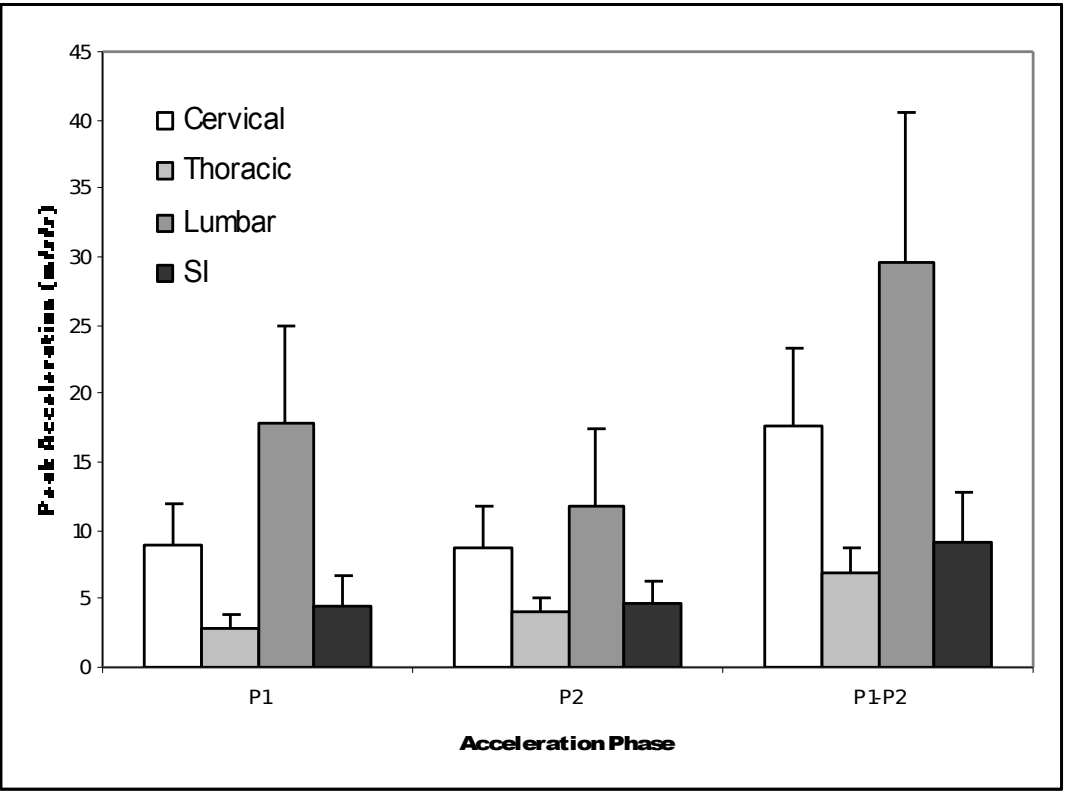


Figure 11. Peak acceleration magnitudes during P1, P2 and P1P2 phases for each spinal level; mean \pm standard deviation.

Spinal level was also a significant factor in determining the temporal parameters of hand acceleration during (figure 12) SMT; P1 peak latency $\left(\mathrm{F}_{3,105}=79.61, \mathrm{p}<0.0001\right)$, P2 peak latency $\left(\mathrm{F}_{3,105}=69.09, \mathrm{p}<0.0001\right)$, and the interval between the P1 and P2 peaks $\left(\mathrm{F}_{3,105}=8.12, \mathrm{p}=0.002\right)$. A general pattern was observed across each acceleration phase with the shortest latencies occurring during lumbar manipulations. Thoracic and cervical manipulations had similar latencies relative to each other and both were significantly longer than those for lumbar SMT $(\mathrm{p}<0.006)$ with the exception of P1 peak latency where the lumbar-cervical pair-wise comparison did not reach statistical significance $(p=0.087)$. Acceleration peak latencies were longest for sacroiliac manipulations for both the P1 and P2 phases ( $<0.0001$ for both) with the exception of the interval between the P1 and P2 peaks which was not different between cervical, thoracic or sacroiliac levels. Another temporal factor, P1 duration, was also significantly related to spinal level $\left(\mathrm{F}_{3,105}=75.20\right.$, $\mathrm{p}<0.0001)$. Subsequent post-hoc tests revealed significant differences between all spinal levels with the following sequence from shortest to longest; lumbar, cervical, thoracic, sacroiliac $(\mathrm{p}<0.05)$.

Temporal acceleration parameters within each spinal level were also assessed for gender differences. No such differences were noted at the cervical, lumbar or SI level. At the thoracic level, a single significant gender difference was present in that P1P2 Lat was greater in males than females $(p=0.048)$. 


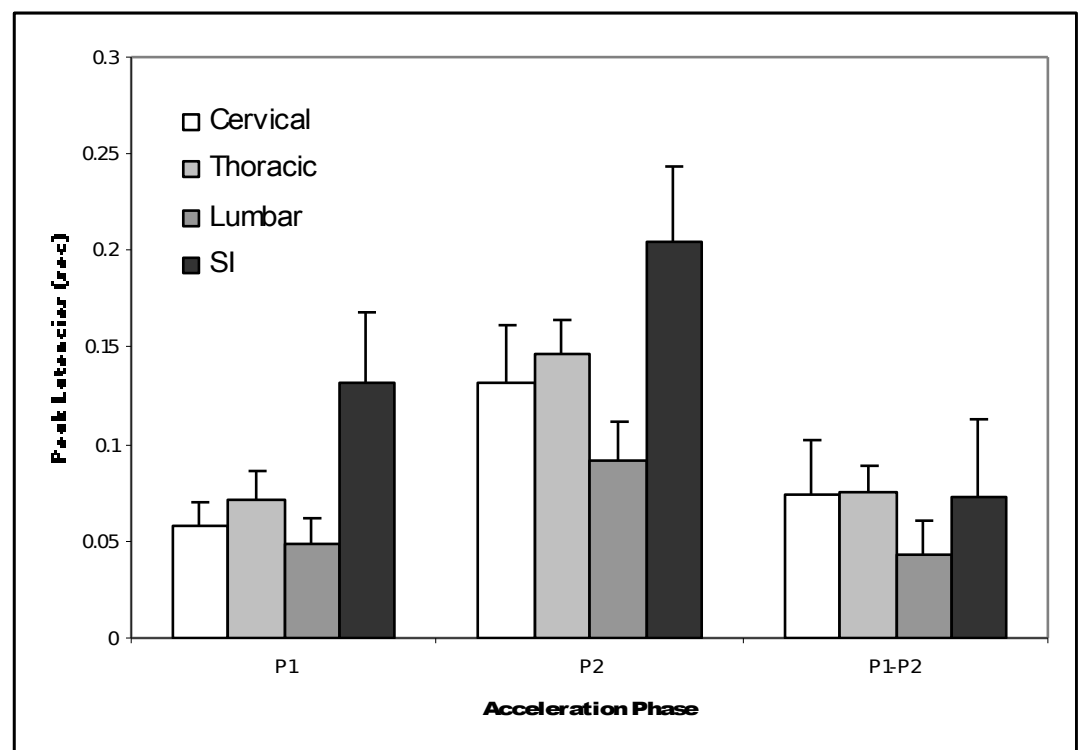

Figure 12. Peak acceleration latencies during $\mathrm{P} 1, \mathrm{P} 2$ and $\mathrm{P} 1 \mathrm{P} 2$ phases for each spinal level; mean \pm standard deviation.

Additional variables assessing the rate of change of acceleration were also analyzed. The slope of the rising portion of P1 was significantly affected by spinal location $\left(\mathrm{F}_{2,81}=31.3, \mathrm{p}<0.0001\right)$. Post-hoc analysis indicated that lumbar manipulations had a greater rising slope compared to all other levels $(\mathrm{p}<0.001)$ and cervical manipulations had greater slopes than those at the thoracic $(\mathrm{p}<0.003)$ level. Lastly, the rate of increase in acceleration (jerk) during the rising phase of P1 was calculated and the peak jerk value during P1 compared across spinal levels. Significant differences were seen between all spinal levels $\left(\mathrm{F}_{2,81}=47.9, \mathrm{p}<0.0001\right)$ with lumbar having the greatest peak jerk values followed by cervical and then thoracic ( $\mathrm{p}<0.001$ for all). Owing to the biphasic P1 segment in sacroiliac trials these were excluded from these analyses due to the lack of a single representative slope or peak jerk value in these trials.

Velocity differences between spinal levels were also examined (Table 2). 
Significant differences for velocity was observed across spinal levels $\left(\mathrm{F}_{1,3}=12.41\right.$, $\mathrm{p}<0.0001)$ with post-hoc tests reporting lumbar SMT having the greatest peak velocities $(p<0.001)$ followed by cervical SMT $(p<0.03)$. Thoracic and SI manipulation had similar peak velocities $(\mathrm{p}>0.9)$.

Table 2. Mean velocities $\left(\mathrm{m} / \mathrm{s}^{2}\right) \pm$ standard deviation for each spinal level

\begin{tabular}{|lcccc|}
\hline & Mean & SD & Min & Max \\
\hline Cervical & 0.414 & 0.14 & 0.051 & 0.630 \\
Thoracic & 0.179 & 0.04 & 0.075 & 0.241 \\
Lumbar & 0.656 & 0.67 & 0.216 & 3.569 \\
SI & 0.188 & 0.07 & 0.068 & 0.355 \\
\hline
\end{tabular}

The above analyses clearly reveal that the SMT acceleration waveforms differ between spinal levels. Specifically, both P1 peak amplitude and latency vary with spinal level. Of interest was whether the differences between magnitudes and latencies occurred independently of one another or whether larger amplitudes were associated with longer latencies. To compare the relationships between these two variables the mean amplitude for each spinal level was plotted against the corresponding mean latency (Figure 13). The plot below shows that larger P1 amplitudes were not associated with longer latencies. For example, the cervical P1 peak amplitude was $218 \%$ greater than that for thoracic SMT. At the same time the cervical P1 peak latency was only $18 \%$ different (lower) than that for thoracic manipulations. These data show that acceleration amplitude and latency can be varied independently during SMT delivered to different spinal levels. 


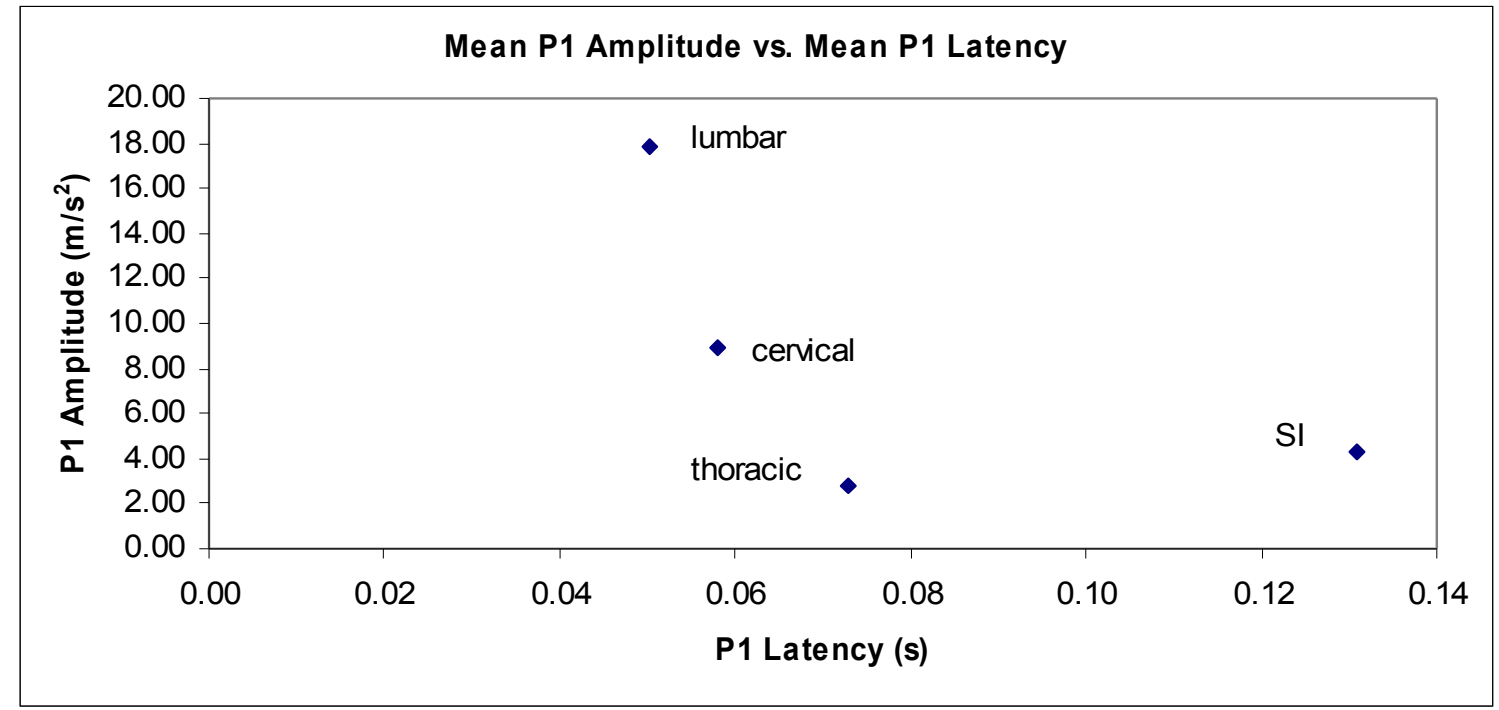

Figure 13. Relationship between $\mathrm{P} 1$ peak acceleration and $\mathrm{P} 1$ peak latency for each spinal level.

Given that different SMT techniques are used to deliver the manipulative thrusts at various spinal levels it may be possible that the differences seen across spinal levels reflect the techniques rather than the inherent interactions of the thrust delivered to the tissue and the response of the tissue to the thrust. To further examine the relationships between SMT acceleration parameters, analyses were conducted within each spinal level which would control for the differences in SMT technique.

\section{Cervical}

At the cervical level, P1 peak amplitude ranged from $1.96 \mathrm{~m} / \mathrm{s}^{2}$ to $15.45 \mathrm{~m} / \mathrm{s}^{2}$ and P1 latency ranged from $23 \mathrm{~ms}$ to $89 \mathrm{~ms}$, however, there was no relationship between P1 amplitude and P1 latency for cervical manipulation $(r=0.05, p=0.766$; Figure 14)

To further examine potential relationships between amplitude and temporal parameters, P1 amplitude and P1 latency were tested for correlations with other 
parameters including $\mathrm{P} 1$ duration, $\mathrm{P} 1$ area under the curve, $\mathrm{P} 2$ amplitude, $\mathrm{P} 2$ latency, P1P2 amplitude and P1P2 latency. As demonstrated in Table 3, P1 amplitude had significant relationships with the other amplitude parameters of P1_AUC, P2 and P1P2 amplitudes. Comparisons of temporal parameters demonstrate significant relationships between P1 latency with P1 duration, P2 latency and P1P2 latency. In addition, P1 latency has a positive relationship with P1 area under the curve. Lastly, and unique to the cervical level, a negative relationship was present between P1 amplitude and both P2 latency $(p=0.027)$ and P1P2 latency $(p=0.011)$.

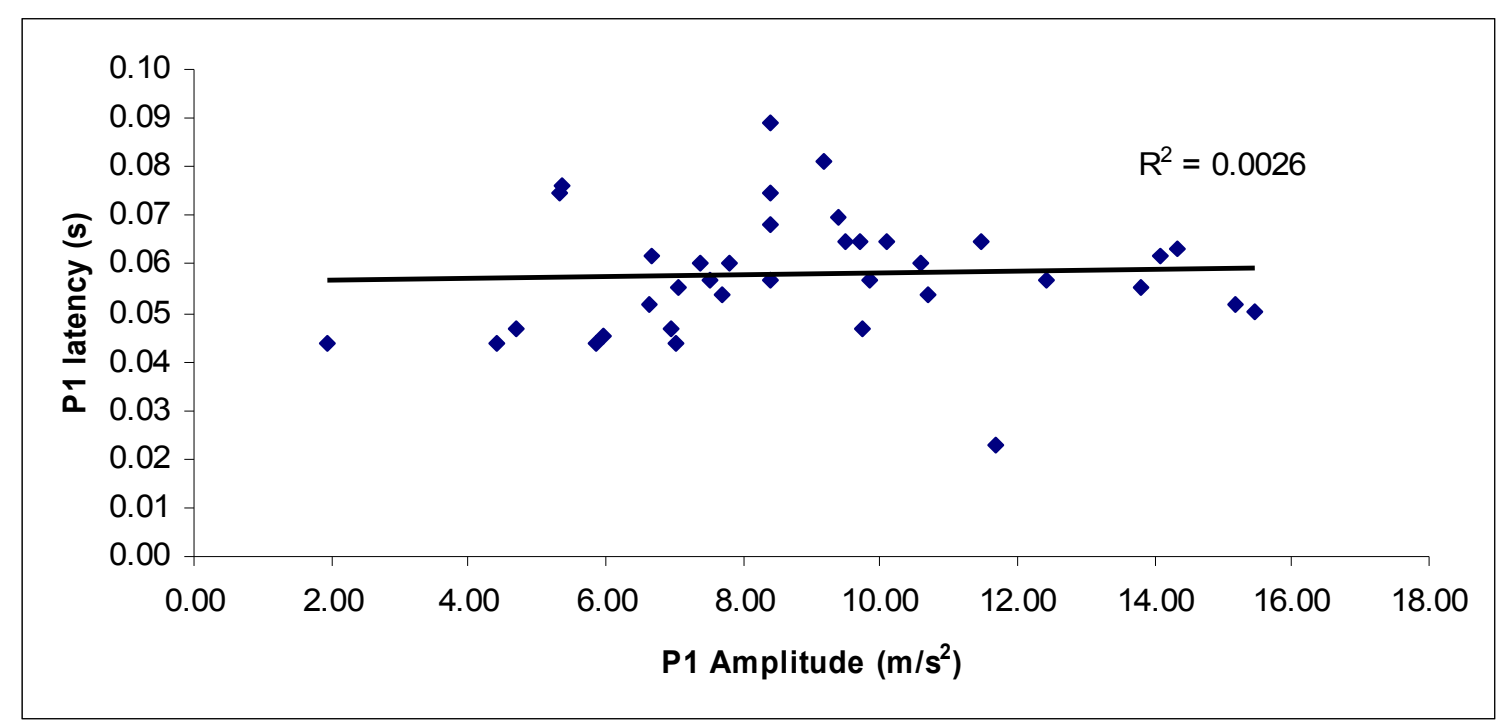

Figure 14. Correlation of $\mathrm{P} 1$ peak amplitude and $\mathrm{P} 1$ peak latency for cervical manipulations.

In an attempt to determine if very early aspects of the acceleration waveform were coupled to later events, correlation coefficients were calculated between the P1 rise slope (P1RS1090) and the main amplitude and temporal parameters of the acceleration waveform. This analysis was also completed using the peak jerk values (Table 4). Significant positive relationships were demonstrated between P1RS1090 and P1, P2 and 
P1P2 amplitudes as well as P1 area under the curve. That is, a steeper P1 rise slope was associated with greater peak amplitudes for P1, P2 and P1P2. Similar to the inverse relationship between P1 peak amplitude and P2 latency reported above, the negative relationship between P1RS1090 and P2 latency occurs only at the cervical spine. Positive correlations were present between peak jerk and P1 amplitude, P1_AUC and P1P2 amplitude while negative correlations were present between Jerk and P2 amplitude and latency. Peak jerk was not related to P1 latency but at the cervical level it does approach significance $(\mathrm{p}=0.052)$. As was the case for the $\mathrm{P} 1$ rise slope, peak jerk was also inversely associated with P2 latency.

Table 3. Pearson correlations ( $\mathrm{r}$ ) between P1 amplitude and P1 latency with the main components for cervical manipulation. Bolded correlations are significant at $\mathrm{p}<0.05$.

\begin{tabular}{|llcrrrrr|}
\hline & & \multicolumn{1}{c}{ P1_DURA P1_AUC } & P2_AMP & P2_LAT & P1P2_AMP & P1P2_LAT \\
\hline P1_AMP & $\mathbf{r}$ & -0.0998 & $\mathbf{0 . 8 6 7 9}$ & $\mathbf{- 0 . 6 9 9 3}$ & $\mathbf{- 0 . 3 6 2 4}$ & $\mathbf{0 . 9 2 5 7}$ & $\mathbf{- 0 . 4 1 4}$ \\
& & $\mathrm{p}=.557$ & $\mathbf{p}=.000$ & $\mathbf{p}<.001$ & $\mathbf{p}=.027$ & $\mathbf{p}<.001$ & $\mathbf{p}=. \mathbf{0 1 1}$ \\
& & & & & & & \\
P1_LAT & $\mathbf{r}$ & $\mathbf{0 . 7 4 5 8}$ & $\mathbf{0 . 3 2 6 1}$ & -0.2786 & $\mathbf{0 . 3 7 7 3}$ & 0.1755 & -0.0519 \\
& & $\mathbf{p}<.001$ & $\mathbf{p}=.049$ & $\mathrm{p}=.095$ & $\mathbf{p}=.021$ & $\mathrm{p}=.299$ & $\mathrm{p}=.760$ \\
\hline
\end{tabular}

Table 4. Pearson correlations (r) between P1RS1090 and Jerk with the main components for cervical manipulation. Bolded correlations are significant at $\mathrm{p}<0.05$.

\begin{tabular}{|llllllllll|}
\hline & & P1_AMP & P1_LAT & P1_DURA & P1_AUC & P2_AMP & P2_LAT & P1P2_AMP & P1P2_LAT \\
\hline P1RS1090 & $\mathbf{r}$ & $\mathbf{0 . 7 6 5 5}$ & -0.4284 & -0.384 & $\mathbf{0 . 4 9 1 5}$ & $-\mathbf{0 . 3 2 9 5}$ & $-\mathbf{0 . 4 1 9 1}$ & $\mathbf{0 . 5 9 9 8}$ & -0.2554 \\
& & $\mathrm{p}<.001$ & $\mathrm{p}=.008$ & $\mathrm{p}=.019$ & $\mathrm{p}=.002$ & $\mathrm{p}=.046$ & $\mathrm{p}=.010$ & $\mathrm{p}<.001$ & $\mathrm{p}=.127$ \\
JERK & & & & & & & & & \\
& $\mathbf{r}$ & $\mathbf{0 . 8 2 1 8}$ & -0.3217 & -0.2806 & $\mathbf{0 . 5 6 2 4}$ & $\mathbf{- 0 . 3 8 8}$ & $\mathbf{- 0 . 4 1 2 5}$ & $\mathbf{0 . 6 6 2}$ & -0.2972 \\
& & $\mathrm{p}<.001$ & $\mathrm{p}=.052$ & $\mathrm{p}=.092$ & $\mathrm{p}<.001$ & $\mathrm{p}=.018$ & $\mathrm{p}=.011$ & $\mathrm{p}<.001$ & $\mathrm{p}=.074$ \\
\hline
\end{tabular}

\section{Thoracic}

P1 amplitude ranged from 0.77 to $4.8 \mathrm{~m} / \mathrm{s}^{2}$ and P1 latency ranged from 45 to 94 ms. Thoracic manipulation did not demonstrate a relationship between P1 amplitude and $\mathrm{P} 1$ latency $(\mathrm{r}=0.18, \mathrm{p}=0.406)$. Therefore, a larger magnitude thoracic manipulation is not associated with a longer P1 latency (Figure 15). 


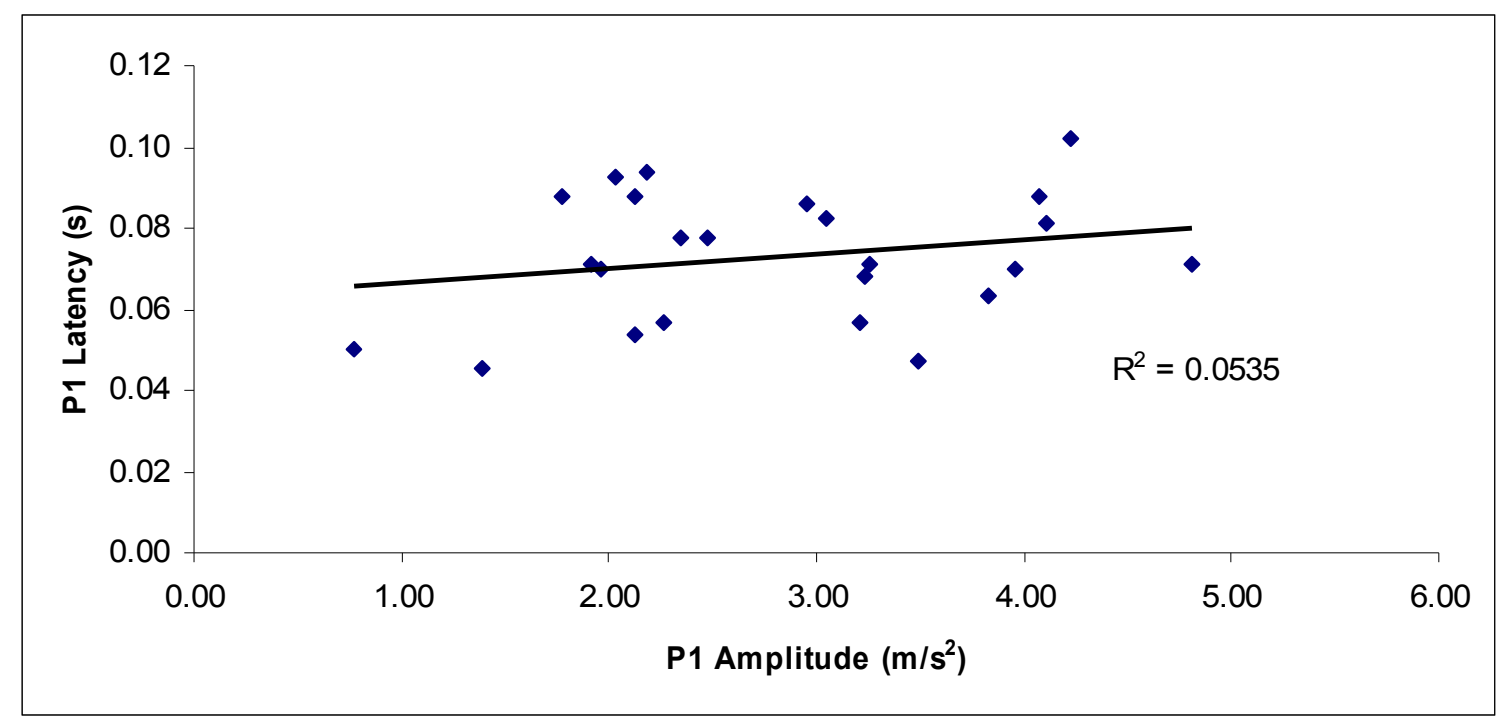

Figure 15. Correlation of $\mathrm{P} 1$ amplitude and $\mathrm{P} 1$ latency for thoracic manipulation.

Within the thoracic manipulations, P1 amplitude had significant positive relationships with P1_AUC and P1P2 amplitude and a significant negative correlation with P2 amplitude while temporal parameters had no relationship with P1 amplitude (Table 5). A significant positive correlation exists between P1 latency with P1 duration and P2 latency but has no relationship with P1P2 latency. Both P1 rise slope (10\%-90\%) and peak jerk had significant positive relationships with P1 amplitude, P1_AUC and P1P2 amplitude and a significant negative relationship with P2 amplitude (Table 6).

Table 5. Pearson correlations (r) between P1 amplitude and P1 latency with the main components for thoracic manipulation. Bolded correlations are significant at $\mathrm{p}<0.05$.

\begin{tabular}{|llcccccc|}
\hline & & P1_DURA P1_AUC & P2_AMP & P2_LAT & P1P2_AMP P1P2_LAT \\
\hline P1_AMP & $\mathbf{r}$ & 0.0575 & $\mathbf{0 . 9 4 1}$ & $\mathbf{- 0 . 8 6 0 5}$ & -0.1126 & $\mathbf{0 . 9 6 6}$ & -0.2394 \\
& & $p=.789$ & $\mathbf{p}<.001$ & $\mathbf{p}<.001$ & $\mathbf{p}=.600$ & $\mathbf{p}<.001$ & $p=.260$ \\
P1_LAT & $\mathbf{r}$ & $\mathbf{0 . 8 7 7 1}$ & 0.2301 & 0.0774 & $\mathbf{0 . 6 8 6 5}$ & 0.0018 & -0.2133 \\
& & $\mathbf{p}<.001$ & $p=.279$ & $p=.719$ & $\mathbf{p}<.001$ & $p=.993$ & $p=.317$ \\
\hline
\end{tabular}


Table 6. Pearson correlations (r) between P1RS1090 and Jerk with the main components for thoracic manipulation. Bolded correlations are significant at $\mathrm{p}<0.05$.

\begin{tabular}{|llcccccccc|}
\hline & & P1_AMP & P1_LAT & P1_DURA & P1_AUC & P2_AMP & P2_LAT & P1P2_AMP & P1P2_LAT \\
\hline P1RS1090 & $\mathbf{r}$ & $\mathbf{0 . 8 1 0 1}$ & -0.3919 & -0.3608 & $\mathbf{0 . 7 1 0 8}$ & $\mathbf{- 0 . 8 1 4 2}$ & -0.3734 & $\mathbf{0 . 8 4 2}$ & -0.0565 \\
& & $\mathbf{p}<.001$ & $\mathrm{p}=.058$ & $\mathrm{p}=.083$ & $\mathbf{p}<.001$ & $\mathbf{p}<.001$ & $\mathrm{p}=.072$ & $\mathbf{p}<.001$ & $\mathrm{p}=.793$ \\
JERK & & & & & & & & & \\
& $\mathbf{r}$ & $\mathbf{0 . 8 7 2 3}$ & -0.2323 & -0.2433 & $\mathbf{0 . 7 6 2 5}$ & $\mathbf{- 0 . 8 4 5 6}$ & -0.3007 & $\mathbf{0 . 8 9 0 9}$ & -0.1402 \\
& & $\mathbf{p}<.001$ & $\mathrm{p}=.275$ & $\mathrm{p}=.252$ & $\mathbf{p}<.001$ & $\mathbf{p}<.001$ & $\mathrm{p}=.153$ & $\mathbf{p}<.001$ & $\mathrm{p}=.514$ \\
\hline
\end{tabular}

\section{Lumbar}

P1 peak amplitude ranged from 7.68 to $40.67 \mathrm{~m} / \mathrm{s}^{2}$ and P1 latency ranged from 32 to $78 \mathrm{~ms}$. P1 amplitude and P1 latency were not correlated $(r=-0.26, p=0.221)$ (Figure 16). P1 amplitude had significant positive relationships with P1_AUC and P1P2 amplitude and a significant negative correlation with P2 amplitude while temporal parameters had no relationship with P1 amplitude (Table 7). A significant positive linear correlation was present between P1 latency and both P1 duration and P2 latency.

Significant positive correlations were present for both P1RS1090 and Jerk with P1 amplitude, P1_AUC and P1P2 amplitude. Significant negative relationships were found between P1RS1090 and both P1 latency and P1 duration and also between Jerk and P1 duration (Table 8). 


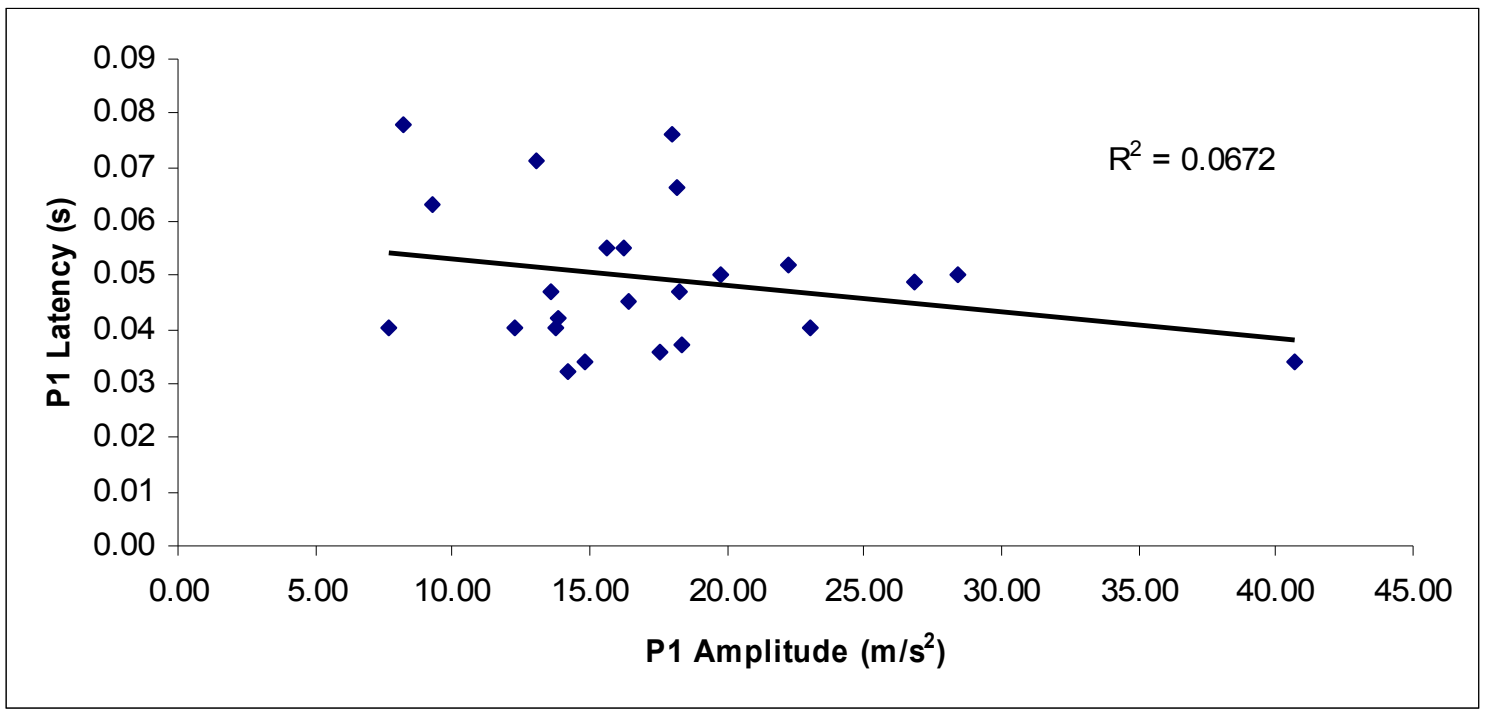

Figure 16. Correlation of $P 1$ amplitude and $P 1$ latency for lumbar manipulation.

Table 7. Pearson correlations (r) between P1 amplitude and P1 latency with the main components for lumbar manipulation. Bolded correlations are significant at $\mathrm{p}<0.05$.

\begin{tabular}{|llcrrrrr|}
\hline & & P1_DURA P1_AUC & P2_AMP & P2_LAT & P1P2_AMP & P1P2_LAT \\
\hline P1_AMP & $\mathbf{r}$ & -0.3151 & $\mathbf{0 . 7 9 2 5}$ & $\mathbf{- 0 . 4 5 8}$ & -0.2914 & $\mathbf{0 . 8 8 4 5}$ & -0.1568 \\
& & $\mathrm{p}=.143$ & $\mathbf{p}<.001$ & $\mathbf{p}=.028$ & $\mathrm{p}=.177$ & $\mathbf{p}<.001$ & $\mathrm{p}=.475$ \\
& & & & & & & \\
P1_LAT & $\mathbf{r}$ & $\mathbf{0 . 8 9 1 7}$ & 0.1009 & 0.0544 & $\mathbf{0 . 4 7 6 2}$ & -0.1678 & -0.2376 \\
& & $\mathbf{p}<.001$ & $\mathrm{p}=.647$ & $\mathrm{p}=.805$ & $\mathbf{p}=.022$ & $\mathrm{p}=.444$ & $\mathrm{p}=.275$ \\
\hline
\end{tabular}

Table 8. Pearson correlations (r) between P1RS1090 and Jerk with the main components for lumbar manipulation. Bolded correlations are significant at $\mathrm{p}<0.05$.

\begin{tabular}{|llcccccccc|}
\hline & & P1_AMP & P1_LAT & P1_DURA & P1_AUC & P2_AMP & P2_LAT & P1P2_AMP & P1P2_LAT \\
\hline P1RS1090 & $\mathbf{r}$ & $\mathbf{0 . 8 6 1 8}$ & $-\mathbf{0 . 5 1 9 5}$ & $\mathbf{- 0 . 5 9 0 3}$ & $\mathbf{0 . 4 7 1 2}$ & -0.2652 & -0.3192 & $\mathbf{0 . 6 9 4 3}$ & 0.0441 \\
& & $\mathbf{p}<.001$ & $\mathbf{p}=.011$ & $\mathbf{p}=.003$ & $\mathbf{p}=.023$ & $\mathbf{p}=.221$ & $\mathbf{p}=.138$ & $\mathbf{p}<.001$ & $\mathrm{p}=.842$ \\
JERK & & & & & & & & & \\
& $\mathbf{r}$ & $\mathbf{0 . 9 1 0 3}$ & -0.3357 & $\mathbf{- 0 . 4 5 9 8}$ & $\mathbf{0 . 5 3 3 6}$ & -0.3716 & -0.2278 & $\mathbf{0 . 7 8 1 4}$ & 0.0047 \\
& & $\mathbf{p}<.001$ & $\mathbf{p}=.117$ & $\mathbf{p}=.027$ & $\mathbf{p}=.009$ & $\mathbf{p}=.081$ & $\mathbf{p}=.296$ & $\mathbf{p}<.001$ & $\mathrm{p}=.983$ \\
\hline
\end{tabular}

\section{Sacroiliac}

P1 peak amplitude ranged from 1.77 to $11.00 \mathrm{~m} / \mathrm{s}^{2}$. P1 latency ranged from 66 to $196 \mathrm{~ms}$. There was no relationship found between the P1 amplitude and P1 latency (r=$0.26, \mathrm{p}=0.196$ ) for sacroiliac manipulation (Figure 17). 


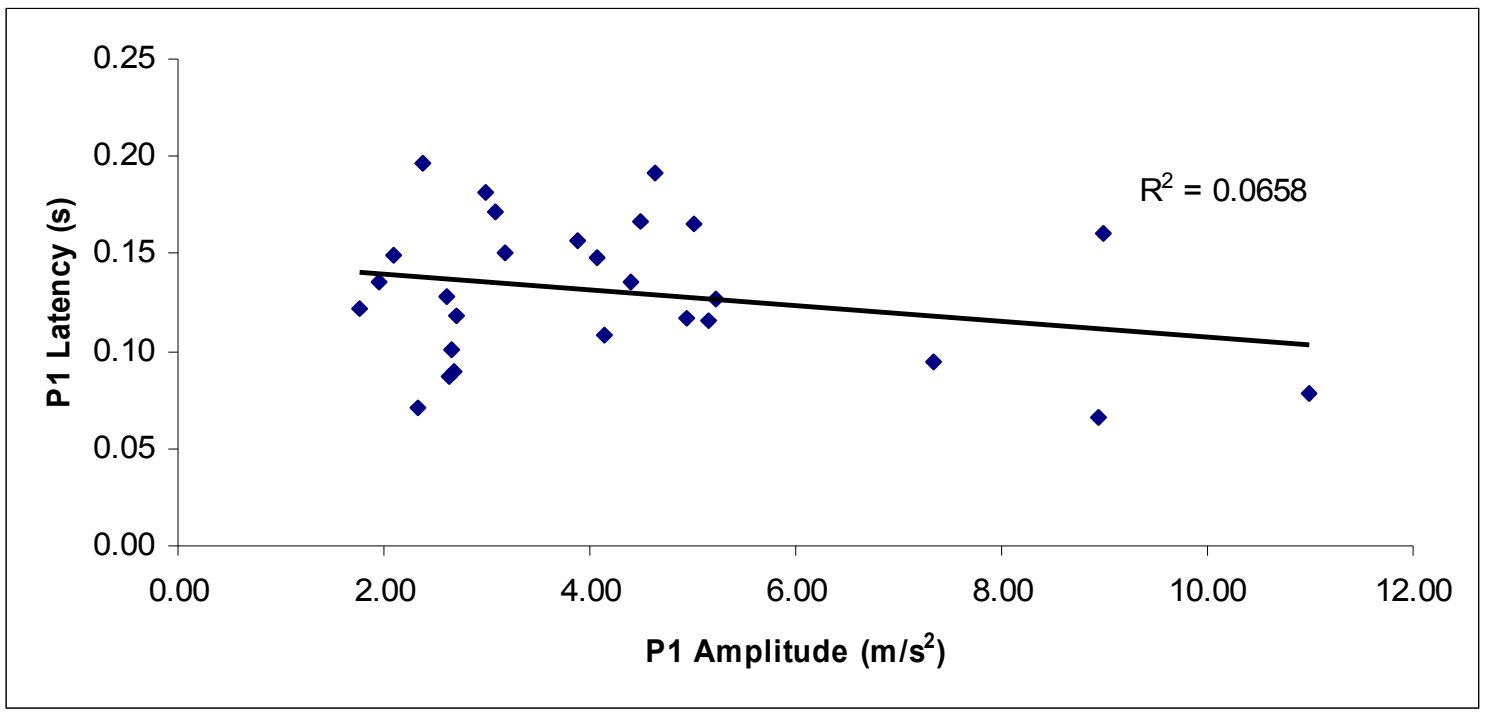

Figure 17. Correlation of $\mathrm{P} 1$ amplitude and $\mathrm{P} 1$ latency for sacroiliac manipulation.

P1 amplitude had significant positive relationships with P1_AUC and P1P2 amplitude and a significant negative correlation with P2 amplitude while temporal parameters had no relationship with P1 amplitude (Table 9). A significant positive correlation exists between P1 latency with P1 duration and P2 latency while a significant linear relationship exists between P1 latency and P1P2 latency.

Table 9. Pearson correlations (r) between P1 amplitude and P1 latency with the main components for sacroiliac manipulation. Bolded correlations are significant at $p<0.05$.

\begin{tabular}{|lccccccc|}
\hline & & P1_DURA P1_AUC & P2_AMP & P2_LAT & P1P2_AMP P1P2_LAT \\
\hline P1_AMP & $\mathbf{r}$ & -0.3537 & $\mathbf{0 . 4 2 8 1}$ & $\mathbf{- 0 . 6 3 3 7}$ & -0.0192 & $\mathbf{0 . 9 3 8 5}$ & 0.2505 \\
& & $\mathrm{p}=.083$ & $\mathbf{p}=. \mathbf{0 3 3}$ & $\mathbf{p}<.001$ & $\mathrm{p}=.927$ & $\mathbf{p}<.001$ & $\mathbf{p}=.227$ \\
P1_LAT & $\mathbf{r}$ & $\mathbf{0 . 8 7 9 2}$ & -0.1574 & 0.1307 & $\mathbf{0 . 4 3 3 4}$ & -0.249 & $\mathbf{- 0 . 5 1 1 2}$ \\
& & $\mathbf{p}<.001$ & $\mathrm{p}=.452$ & $\mathrm{p}=.533$ & $\mathbf{p}=. \mathbf{0 3 0}$ & $\mathrm{p}=.230$ & $\mathbf{p}=.009$ \\
\hline
\end{tabular}

\section{Correlation of Patient Anthropometric Parameters with SMT Characteristics}

Univariate correlation analyses were used to investigate whether patient anthropometric features were associated with various features of the acceleration waveform recorded during the manipulations. Given the overall low level of correlations, 
especially for body mass and BMI, partial correlations were also completed for P1

amplitude to control for potential gender effects.

\section{Cervical}

The anthropometric features of the 21 patients receiving cervical SMT trials are listed in Table 10 along with the acceleration parameters.

Table 10. Descriptive statistics of components for cervical SMT trials.

\begin{tabular}{|c|c|c|c|c|}
\hline Variables & Mean & SD & Min & Max \\
\hline $\begin{array}{l}\text { Height }(\mathrm{m}) \\
\text { Body Mass (kg) }\end{array}$ & $\begin{array}{c}1.650 \\
80.250\end{array}$ & $\begin{array}{c}0.100 \\
17.650\end{array}$ & $\begin{array}{c}1.473 \\
47.800\end{array}$ & $\begin{array}{c}1.880 \\
111.400\end{array}$ \\
\hline BMI $\left(\mathrm{kg} / \mathrm{m}^{2}\right)$ & 29.170 & 5.170 & 20.581 & 38.178 \\
\hline Age (yrs) & 48.730 & 10.180 & 30.000 & 69.000 \\
\hline P1 Amplitude $\left(\mathrm{m} / \mathrm{s}^{2}\right)$ & 8.892 & 3.152 & 1.957 & 15.448 \\
\hline P1 Latency (s) & 0.058 & 0.013 & 0.023 & 0.089 \\
\hline P1 Duration (s) & 0.084 & 0.017 & 0.050 & 0.138 \\
\hline P1_AUC & 0.335 & 0.103 & 0.057 & 0.577 \\
\hline P2 Amplitude $\left(\mathrm{m} / \mathrm{s}^{2}\right)$ & -8.742 & 3.001 & -16.126 & -2.280 \\
\hline P2 Latency (s) & 0.132 & 0.029 & 0.083 & 0.198 \\
\hline P1P2 Amplitude (m/s²) & 17.635 & 5.672 & 4.237 & 31.574 \\
\hline P1P2 Latency (s) & 0.074 & 0.027 & 0.034 & 0.154 \\
\hline P1RS1090 & 227.423 & 121.145 & 54.126 & 715.150 \\
\hline Jerk $\left(\mathrm{m} / \mathrm{s}^{3}\right)$ & 329.529 & 163.579 & 98.554 & 841.523 \\
\hline Peak Velocity $\left(\mathrm{m} / \mathrm{s}^{2}\right)$ & 0.414 & 0.14 & 0.051 & 0.630 \\
\hline
\end{tabular}

Table 11. Pearson correlations (r) of patient anthropometric parameters with cervical SMT main components and subcomponents. Correlations are significant if $\mathrm{p}<0.05$.

\begin{tabular}{|c|c|c|c|c|c|c|c|c|c|c|c|}
\hline \multicolumn{3}{|c|}{ Height } & \multicolumn{3}{|c|}{ Body Mass } & \multicolumn{3}{|c|}{ BMI } & \multicolumn{3}{|c|}{ AGE } \\
\hline P1_AMP & -0.2352 & $p=.161$ & P1_AMP & 0.098 & $p=564$ & P1_AMP & 0.3334 & $p=044$ & P1_AMP & 0.3954 & $p=015$ \\
\hline P1_LAT & 0.2885 & $p=0.083$ & P1_LAT & 0.3272 & $\mathrm{p}=0.048$ & P1_LAT & 0.1845 & $p=.274$ & P1_LAT & -0.0071 & $p=.967$ \\
\hline P1_DURA & 0.3172 & $p=056$ & P1_DURA & 0.2422 & $p=149$ & P1_DURA & 0.0724 & $p=.670$ & P1_DURA & -0.0129 & $p=$ \\
\hline P1_AUC & -0.1269 & $p=.454$ & P1_AUC & 0.0933 & $p=583$ & P1_AUC & 0.2391 & $p=.154$ & P1_AUC & 0.3884 & $p=018$ \\
\hline P2_AMP & 0.1166 & $p=492$ & P2_AMP & -0.0903 & $p=595$ & P2_AMP & -0.229 & $p=.173$ & P2_AMP & -0.2025 & $p=.229$ \\
\hline P2_LAT & 0.3495 & $p=0.034$ & P2_LAT & 0.1216 & $p=474$ & P2_LAT & -0.0912 & $p=591$ & P2_LAT & -0.1294 & $p=.445$ \\
\hline P1P2_AMP & -0.1924 & $p=.254$ & P1P2_AMP & 0.1022 & $p=547$ & P1P2_AMP & 0.3064 & $p=.065$ & P1P2_AMP & 0.3269 & $p=0.048$ \\
\hline P1P2_LAT & 0.2445 & $p=145$ & P1P2_LAT & -0.019 & $p=.911$ & P1P2_LAT & -0.183 & $p=.278$ & P1P2_LAT & -0.1363 & $p=.421$ \\
\hline P1RS1090 & -0.3653 & $p=0.026$ & P1RS1090 & -0.0063 & $p=.970$ & P1RS1090 & 0.2937 & $p=.078$ & P1RS1090 & 0.2448 & $p=.144$ \\
\hline JERK & -0.3007 & $p=071$ & JERK & 0.0026 & $p=988$ & JERK & 0.2656 & $p=.112$ & JERK & 0.3491 & $p=0.034$ \\
\hline
\end{tabular}




\section{Height}

The overall trend present in the cervical SMT trials was that longer acceleration latencies were observed in taller patients. This was indicated by the positive relationships present between height and several temporal measures of the cervical SMT trials including P1 latency $(\mathrm{p}=0.083)$, P2 latency $(\mathrm{p}=0.034)$ and total P1 duration $(\mathrm{p}=0.056)$. Despite the indication of longer latencies in taller patients there was no increase in the magnitude of the SMT events in taller patients as P1 amplitude ( $\mathrm{p}=0.161), \mathrm{P} 2$ amplitude $(\mathrm{p}=0.492)$ and $\mathrm{P} 1 \mathrm{P} 2$ amplitude $(\mathrm{p}=0.254)$ were not related to patient height. $\mathrm{P} 1$ rise slope and peak jerk were negatively associated with patient height (P1RS1090, $\mathrm{p}=0.026$; jerk, $\mathrm{p}=0.071$ ). Given the known relationships between anthropometric measures and gender, partial correlations controlling for gender were performed for the primary outcome of P1 amplitude. Although the zero order correlation was not significant (Table 10), a significant partial correlation was present between height and P1 amplitude $(r=-0.480$, $p=0.021)$ when gender was factored into the analysis.

\section{Body Mass}

The magnitude of cervical manipulations was unrelated to body mass in that P1 peak amplitude $(\mathrm{p}=0.564), \mathrm{P} 2$ peak amplitude $(\mathrm{p}=0.595)$ and P1P2 peak amplitude $(\mathrm{p}=0.547)$ were not significantly correlated with body mass. Similarly, P1 duration $(\mathrm{p}=0.149)$ and P1_AUC $(\mathrm{p}=0.583)$ were not related to body mass. P1 peak latency was positively correlated with body mass $(\mathrm{p}=0.048)$ indicating that, within cervical manipulations, P1 peaks at a later time for heavier patients. However, no other temporal parameter showed a similar relationship. Despite the large contribution of gender to the 
height-P1 amplitude relationship, gender did not influence the correlation between body mass and P1 amplitude ( $r=0.098$ vs. $r=0.072, \mathrm{p}>0.56$ for both).

\section{BMI}

A significant positive linear relationship was found between BMI and P1 amplitude ( $\mathrm{p}=0.044)$ indicating that larger acceleration magnitudes were observed in patients with a higher BMI. This relationship is further supported by the positive correlation between BMI and the P1 rise slope (P1RS1090, $\mathrm{p}=0.078)$. These larger magnitude events occur without a change in the temporal parameters of the P1 phase. The P1 latency $(\mathrm{p}=0.274)$ and $\mathrm{P} 1$ duration $(\mathrm{p}=0.670)$ had no relationship with BMI. Moreover, there is no relationship of BMI to P2 latency ( $\mathrm{p}=0.591)$. For the most part, the temporal aspects of cervical SMT appear to be relatively constant despite the positive linear relationship between BMI and P1 amplitude. Similar to body mass, controlling for gender did not alter the correlation between BMI and P1amplitude.

Age

The acceleration magnitudes of cervical manipulation were larger in older subjects as indicated by positive correlations between age and P1 peak amplitude $(\mathrm{p}=0.015)$ and $\mathrm{P} 1 \_\mathrm{AUC}(\mathrm{p}=0.018)$. In contrast, $\mathrm{P} 1$ latency $(\mathrm{p}=0.967)$ and $\mathrm{P} 1$ duration $(p=0.940)$ had no relationship with age. Partial correlation showed no impact of gender on the age-P1 amplitude relationship.

\section{Thoracic}

The anthropometric features of the 24 patients receiving thoracic manipulation are listed in Table 12 along with acceleration parameters. 
Table 12. Descriptive statistics of components for thoracic SMT trials.

\begin{tabular}{|lcccc|}
\hline \multicolumn{1}{|c}{ Variables } & Mean & SD & Min & Max \\
\hline Height (m) & 1.654 & 0.100 & 1.473 & 1.880 \\
Body Mass (kg) & 78.063 & 18.098 & 47.800 & 111.400 \\
BMI (kg/m ${ }^{2}$ ) & 28.327 & 5.040 & 20.581 & 38.178 \\
Age (yrs) & 49.042 & 12.038 & 29.000 & 75.000 \\
\hline P1 Amplitude (m/s ${ }^{2}$ ) & 2.804 & 1.010 & 0.769 & 4.804 \\
P1 Latency (s) & 0.071 & 0.015 & 0.045 & 0.094 \\
P1 Duration (s) & 0.102 & 0.013 & 0.079 & 0.128 \\
P1_AUC & 0.154 & 0.053 & 0.040 & 0.238 \\
P2 Amplitude (m/s ${ }^{2}$ ) & -4.086 & 0.967 & -5.672 & -2.288 \\
P2 Latency (s) & 0.147 & 0.017 & 0.115 & 0.183 \\
P1P2 Amplitude (m/s ${ }^{2}$ ) & 6.891 & 1.907 & 3.057 & 10.476 \\
P1P2 Latency (s) & 0.076 & 0.013 & 0.057 & 0.110 \\
\hline P1RS1090 & 52.351 & 24.493 & 22.257 & 100.374 \\
Jerk (m/s ${ }^{3}$ ) & 79.522 & 33.977 & 32.685 & 141.820 \\
\hline Peak Velocity (m/s ${ }^{2}$ ) & 0.179 & 0.043 & 0.075 & 0.241 \\
Peak Velocity Latency (s) & 0.103 & 0.012 & 0.084 & 0.128 \\
Peak Displacement (m) & 12.227 & 3.022 & 4.481 & 17.257 \\
Peak Displacement Latency & 0.158 & 0.015 & 0.131 & 0.188 \\
\hline
\end{tabular}

Table 13. Pearson correlations (r) of patient anthropometric parameters with thoracic SMT main components and subcomponents. Correlations are significant if $\mathrm{p}<0.05$.

\begin{tabular}{|c|c|c|c|c|c|c|c|c|c|c|c|}
\hline \multicolumn{3}{|c|}{ Height } & \multicolumn{3}{|c|}{ Body Mass } & \multicolumn{3}{|c|}{ BMI } & \multicolumn{3}{|c|}{ AGE } \\
\hline P1_AMP & -0.163 & $p=.447$ & P1_AMP & 0.1441 & $p=502$ & P1_AMP & 0.3309 & $p=.114$ & P1_AMP & 0.0349 & $p=872$ \\
\hline P1_LAT & 0.0284 & $p=.895$ & P1_LAT & 0.1383 & $p=519$ & P1_LAT & 0.1886 & $p=377$ & P1_LAT & -0.1462 & $p=.496$ \\
\hline P1_DURA & 0.1831 & $p=392$ & P1_DURA & 0.1448 & $p=500$ & P1_DURA & 0.0987 & $p=646$ & P1_DURA & -0.1637 & $p=.445$ \\
\hline P1_AUC & -0.0325 & $p=.880$ & P1_AUC & 0.2531 & $p=.233$ & P1_AUC & 0.3863 & $p=0.062$ & P1_AUC & -0.091 & $p=672$ \\
\hline P2_AMP & 0.1961 & $p=358$ & P2_AMP & -0.1056 & $p=623$ & P2_AMP & -0.2899 & $p=169$ & P2_AMP & 0.0247 & $p=.909$ \\
\hline P2_LAT & 0.3102 & $p=140$ & P2_LAT & 0.1182 & $p=582$ & P2_LAT & -0.0325 & $p=.880$ & P2 LAT & -0.0446 & $p=836$ \\
\hline P1P2_AMP & -0.1858 & $p=385$ & P1P2_AMP & 0.1299 & $p=545$ & P1P2_AMP & 0.3223 & $p=125$ & P1P2_AMP & 0.0059 & $p=.978$ \\
\hline P1P2_LAT & 0.3846 & $p=.064$ & P1P2_LAT & 0.0018 & $p=.993$ & P1P2_LAT & -0.2579 & $p=.224$ & P1P2_LAT & 0.106 & $p=622$ \\
\hline P1RS1090 & -0.0759 & $p=725$ & P1RS1090 & 0.0852 & $p=692$ & P1RS1090 & 0.1748 & $p=.414$ & P1RS1090 & -0.0357 & $p=869$ \\
\hline JERK & -0.1145 & $p=594$ & JERK & 0.1417 & $p=.509$ & JERK & 0.281 & $p=183$ & JERK & 0.0254 & $p=.906$ \\
\hline
\end{tabular}

\section{Height}

There was no relationship between height and P1 amplitude ( $\mathrm{p}=0.447), \mathrm{P} 1$ latency

$(\mathrm{p}=0.895), \mathrm{P} 1$ duration $(\mathrm{p}=0.392)$ or $\mathrm{P} 1 \_\mathrm{AUC}(\mathrm{p}=0.880)$. Similarly, there was no 
relationship between height and P2 amplitude $(\mathrm{p}=.358)$ or P2 latency $(\mathrm{p}=.140)$. Partial correlation showed no effect of gender on the height-P1 amplitude relationship ( $\mathrm{p}>0.45)$.

\section{Body Mass}

Body mass also had no relationship with any thoracic SMT variable (P1 amplitude $(\mathrm{p}=0.502), \mathrm{P} 1$ latency $(\mathrm{p}=0.519), \mathrm{P} 1$ duration $(\mathrm{p}=0.500)$ and P1_AUC $(p=0.203)$. The contribution of gender to the body mass-P1 amplitude relationship did not influence the correlation between body mass and $\mathrm{P} 1$ amplitude $(\mathrm{r}=.144$ vs. $\mathrm{r}=.230, \mathrm{p}>0.32$ for both)

BMI

BMI showed little relationship with SMT parameters although a weak trend toward greater magnitudes with increasing BMI was seen for P1 ( $\mathrm{p}=0.114), \mathrm{P} 1 \_$AUC $(p=0.062), P 2(p=0.169)$, and P1P2 $(p=0.125)$. No such trends were seen in temporal parameters (P1 latency, $\mathrm{p}=0.377 ; \mathrm{P} 2$ latency, $\mathrm{p}=0.880 ; \mathrm{P} 1 \mathrm{P} 2$ latency, $\mathrm{p}=0.224)$. Similar to body mass, controlling for gender did not alter the correlation between BMI and Plamplitude.

Age

There was no relationship between age and P1 amplitude ( $p=0.872), P 1$ latency $(p=0.496), P 1$ duration $(p=0.445)$ and $P 1 \_A U C(p=0.672)$ or any other aspect of thoracic SMT. Partial correlation showed no impact of gender on the age-P1 amplitude relationship.

\section{Lumbar}

The anthropometric features of the 23 patients receiving lumbar manipulation are 
listed in Table 14 along with acceleration parameters.

Table 14. Descriptive statistics of components for lumbar SMT trials.

\begin{tabular}{|c|c|c|c|c|}
\hline Variables & Mean & SD & Min & Max \\
\hline Height (m) & 1.672 & 0.103 & 1.473 & 1.880 \\
\hline Body Mass (kg) & 83.513 & 17.046 & 47.800 & 111.400 \\
\hline BMI $\left(\mathrm{kg} / \mathrm{m}^{2}\right)$ & 29.775 & 5.218 & 20.581 & 41.129 \\
\hline Age (yrs) & 47.458 & 11.390 & 29.000 & 69.000 \\
\hline P1 Amplitude (m/s $\left.{ }^{2}\right)$ & 17.865 & 7.060 & 7.684 & 40.666 \\
\hline P1 Latency (s) & 0.049 & 0.013 & 0.032 & 0.078 \\
\hline P1 Duration (s) & 0.069 & 0.013 & 0.050 & 0.092 \\
\hline P1_AUC & 0.569 & 0.198 & 0.287 & 1.015 \\
\hline P2 Amplitude (m/s $\left.{ }^{2}\right)$ & -11.674 & 5.753 & -30.571 & -5.580 \\
\hline P2 Latency (s) & 0.092 & 0.019 & 0.066 & 0.135 \\
\hline P1P2 Amplitude $\left(\mathrm{m} / \mathrm{s}^{2}\right)$ & 29.538 & 10.961 & 14.412 & 58.991 \\
\hline P1P2 Latency (s) & 0.043 & 0.017 & 0.024 & 0.089 \\
\hline P1RS1090 & 501.145 & 341.700 & 103.632 & 1856.167 \\
\hline Jerk $\left(\mathrm{m} / \mathbf{s}^{3}\right)$ & 790.611 & 436.714 & 288.770 & 2403.847 \\
\hline Peak Velocity $\left(\mathrm{m} / \mathrm{s}^{2}\right)$ & 0.440 & 0.97 & -1.883 & 3.569 \\
\hline
\end{tabular}

Table 15. Pearson correlations (r) of patient anthropometric parameters with lumbar SMT main components and subcomponents. Correlations are significant if $\mathrm{p}<0.05$.

\begin{tabular}{|c|c|c|c|c|c|c|c|c|c|c|c|}
\hline \multicolumn{3}{|c|}{ Height } & \multicolumn{3}{|c|}{ Body Mass } & \multicolumn{3}{|c|}{ BMI } & \multicolumn{3}{|c|}{ AGE } \\
\hline P1_AMP & 0.3603 & $p=091$ & P1_AMP & 0.1968 & $p=368$ & P1_AMP & -0.0091 & $p=.967$ & P1_AMP & 0.125 & $p=.570$ \\
\hline P1_LAT & 0.154 & $p=.483$ & P1_LAT & -0.0261 & $p=.906$ & P1_LAT & -0.1104 & $p=616$ & P1_LAT & -0.2269 & $p=298$ \\
\hline P1_DURA & 0.1705 & $p=.437$ & P1_DURA & 0.0021 & $p=.993$ & P1_DURA & -0.0927 & $p=674$ & P1_DURA & -0.2561 & $p=.238$ \\
\hline P1_AUC & 0.4898 & $p=.018$ & P1_AUC & 0.2568 & $p=.237$ & P1_AUC & -0.0101 & $p=.963$ & P1_AUC & 0.1104 & $p=616$ \\
\hline P2_AMP & -0.5112 & $p=.013$ & P2_AMP & -0.1956 & $p=371$ & P2_AMP & 0.1264 & $p=.566$ & P2_AMP & 0.0085 & $p=.969$ \\
\hline P2_LAT & -0.1363 & $p=535$ & P2_LAT & -0.1348 & $p=540$ & P2_LAT & -0.0454 & $p=.837$ & P2_LAT & -0.1397 & $p=.525$ \\
\hline P1P2_AMP & 0.5003 & $p=.015$ & P1P2_AMP & 0.2294 & $p=.292$ & P1P2_AMP & -0.0722 & $p=.743$ & P1P2_AMP & 0.0761 & $p=.730$ \\
\hline P1P2_LAT & -0.2682 & $p=.216$ & P1P2_LAT & -0.129 & $p=.558$ & P1P2_LAT & 0.0342 & $p=.877$ & P1P2_LAT & 0.0189 & $p=.932$ \\
\hline P1RS1090 & 0.1084 & $p=623$ & P1RS1090 & 0.0654 & $p=.767$ & P1RS1090 & -0.008 & $p=.971$ & P1RS1090 & 0.2109 & $p=.334$ \\
\hline JERK & 0.2051 & $p=348$ & JERK & 0.1016 & $p=644$ & JERK & -0.0226 & $p=.919$ & JERK & 0.1393 & $p=526$ \\
\hline
\end{tabular}

\section{Height}

P1 acceleration amplitude tended to be larger in patients of greater height $(\mathrm{p}=0.091)$. Consistent with this, a significant positive relationship was found between height and the P1 area under the curve $(\mathrm{p}=0.018)$. In contrast, no trends were observed 
between the temporal parameters of lumbar SMT and patient height including P1 latency $(\mathrm{p}=0.483)$ and $\mathrm{P} 1$ duration $(\mathrm{p}=0.437)$.

Height had a significant negative linear relationship with P2 amplitude ( $\mathrm{p}=0.013)$, indicating that P2 amplitudes were of a greater magnitude (more negative) in taller subjects. In keeping with the separate relationships between height and P1 amplitude and height and P2 amplitude, height was also positively associated with a larger P1P2 amplitude $(\mathrm{p}=0.015)$. No relationships were present between patient height and temporal parameters of the P2 phase of lumbar SMT. A trend was present between height and P1 amplitude ( $\mathrm{p}=0.091$ ), however, adjusting for gender completely removed this trend ( $>0.60)$ but did not remove the significant relationship between height and P2 amplitude.

\section{Body Mass}

Body mass had no relationship with any of the lumbar SMT characteristics including P1 amplitude ( $\mathrm{p}=0.368), \mathrm{P} 1$ latency $(\mathrm{p}=0.906), \mathrm{P} 1$ duration $(\mathrm{p}=0.993)$ and P1_AUC $(\mathrm{p}=0.237)$. Gender did not influence the correlation between body mass and P1 amplitude $(\mathrm{p}<0.98)$.

\section{BMI}

P1 amplitude ( $\mathrm{p}=0.967), \mathrm{P} 1$ latency $(\mathrm{p}=0.616), \mathrm{P} 1$ duration $(\mathrm{p}=0.674), \mathrm{P} 1 \_\mathrm{AUC}$ $(\mathrm{p}=0.963)$ and all other parameters of lumbar SMT were not correlated with BMI. Similar to body mass, controlling for gender did not alter the correlation between BMI and P1amplitude.

\section{Age}


No relationships between age and lumbar SMT characteristics were

observed; P1 amplitude ( $p=0.570), P 1$ duration $(p=0.238), P 1$ latency $(p=0.298)$, P1_AUC $(\mathrm{p}=0.616)$. Gender did not influence the correlation between age and P1 amplitude.

\section{Sacroiliac}

The anthropometric features of the 25 patients receiving sacroiliac manipulation are listed in Table 16 along with acceleration parameters.

Table 16. Descriptive statistics of components for sacroiliac SMT trials.

\begin{tabular}{|c|c|c|c|c|}
\hline Variables & Mean & SD & Min & Max \\
\hline $\begin{array}{l}\text { Height }(\mathrm{m}) \\
\text { Body Mass (kg) }\end{array}$ & $\begin{array}{c}1.669 \\
82.326\end{array}$ & $\begin{array}{c}0.100 \\
17.042\end{array}$ & $\begin{array}{c}1.473 \\
47.800\end{array}$ & $\begin{array}{c}1.880 \\
111.400\end{array}$ \\
\hline $\begin{array}{l}\text { BMI }\left(\mathrm{kg} / \mathrm{m}^{2}\right) \\
\text { Age (yrs) }\end{array}$ & $\begin{array}{l}29.419 \\
48.259\end{array}$ & $\begin{array}{c}5.117 \\
11.302\end{array}$ & $\begin{array}{l}20.581 \\
29.000\end{array}$ & $\begin{array}{l}41.129 \\
69.000\end{array}$ \\
\hline $\begin{array}{l}\text { P1 Amplitude }\left(\mathrm{m} / \mathrm{s}^{2}\right) \\
\text { P1 Latency }(\mathrm{s}) \\
\text { P1 Duration (s) } \\
\text { P1_AUC }\end{array}$ & $\begin{array}{l}4.376 \\
0.132 \\
0.154 \\
0.150\end{array}$ & $\begin{array}{l}2.405 \\
0.037 \\
0.036 \\
0.053\end{array}$ & $\begin{array}{l}1.769 \\
0.066 \\
0.079 \\
0.065\end{array}$ & $\begin{array}{l}10.996 \\
0.196 \\
0.227 \\
0.271\end{array}$ \\
\hline $\begin{array}{l}\text { P2 Amplitude }\left(\mathrm{m} / \mathrm{s}^{2}\right) \\
\text { P2 Latency }(\mathrm{s})\end{array}$ & $\begin{array}{r}-4.681 \\
0.205\end{array}$ & $\begin{array}{l}1.636 \\
0.038\end{array}$ & $\begin{array}{l}-9.205 \\
0.141\end{array}$ & $\begin{array}{c}-1.933 \\
0.282\end{array}$ \\
\hline $\begin{array}{l}\text { P1P2 Amplitude }\left(\mathrm{m} / \mathrm{s}^{2}\right) \\
\text { P1P2 Latency }(\mathrm{s})\end{array}$ & $\begin{array}{l}9.057 \\
0.073\end{array}$ & $\begin{array}{l}3.667 \\
0.040\end{array}$ & $\begin{array}{l}4.564 \\
0.021\end{array}$ & $\begin{array}{c}20.201 \\
0.194\end{array}$ \\
\hline Peak Velocity $\left(\mathrm{m} / \mathrm{s}^{2}\right)$ & 0.188 & 0.07 & 0.068 & 0.355 \\
\hline
\end{tabular}

Table 17. Pearson correlations (r) of patient anthropometric parameters with sacroiliac SMT main components and subcomponents. Correlations are significant if $\mathrm{p}<0.05$.

\begin{tabular}{|c|c|c|c|c|c|c|c|c|c|c|c|}
\hline \multicolumn{3}{|c|}{ Height } & \multicolumn{3}{|c|}{ Body Mass } & \multicolumn{3}{|c|}{ BMI } & \multicolumn{3}{|c|}{ AGE } \\
\hline P1_AMP & 0.1719 & $p=.411$ & P1_AMP & 0.4535 & $p=023$ & P1_AMP & 0.4567 & $p=.022$ & P1_AMP & 0.0831 & $p=693$ \\
\hline P1_LAT & -0.016 & $p=.940$ & P1_LAT & 0.0515 & $p=.807$ & P1_LAT & 0.0483 & $p=.819$ & P1__LAT & -0.3103 & $p=.131$ \\
\hline P1_DURA & 0.0894 & $p=671$ & P1_DURA & 0.1771 & $p=397$ & P1_DURA & 0.1366 & $p=.515$ & P1_DURA & -0.2038 & $p=329$ \\
\hline P1_AUC & 0.2785 & $p=.178$ & P1_AUC & 0.0698 & $p=.740$ & P1_AUC & -0.1169 & $p=.578$ & P1_AUC & 0.1732 & $p=408$ \\
\hline P2_AMP & 0.1442 & $p=492$ & P2_AMP & 0.0583 & $p=782$ & P2_AMP & -0.0429 & $p=.839$ & P2_AMP & -0.1964 & $p=347$ \\
\hline P2_LAT & 0.0105 & $p=.960$ & P2_LAT & 0.17 & $p=.416$ & P2_LAT & 0.1785 & $p=393$ & P2__LAT & 0.0399 & $p=.850$ \\
\hline P1P2_AMP & 0.0484 & $p=.818$ & P1P2_AMP & 0.2714 & $p=.189$ & P1P2_AMP & 0.3187 & $p=.121$ & P1P2_AMP & 0.1421 & $p=.498$ \\
\hline P1P2_LAT & 0.0247 & $p=.907$ & P1P2_LAT & 0.1146 & $p=586$ & P1P2_LAT & 0.1255 & $p=.550$ & P1P2_LAT & 0.3249 & $p=113$ \\
\hline
\end{tabular}




\section{Height}

Neither P1 amplitude $(p=0.411), P 1$ latency $(p=0.940), P 1$ duration $(p=0.671)$ nor area under the curve $\left(\mathrm{P} 1 \_\mathrm{AUC}, \mathrm{p}=0.178\right)$ had any relationship with height. The relationship between height and P1 amplitude was not significant after controlling for gender $(\mathrm{p}>0.30)$.

\section{Body Mass}

P1 amplitude $(p=0.023)$ and Jerk $(p=0.015)$ were positively correlated with body mass. In contrast, $\mathrm{P} 1$ latency $(\mathrm{p}=0.807), \mathrm{P} 1$ duration $(\mathrm{P}=0.397)$ and $\mathrm{P} 1 \_\mathrm{AUC}(\mathrm{p}=0.740)$ had no correlations with body mass. Controlling for gender weakened the relationship between body mass and P1 amplitude (p-value increased from 0.023 to 0.083 ).

\section{BMI}

P1 amplitude $(\mathrm{p}=0.022)$ and peak jerk $(\mathrm{p}=0.007)$ were positively correlated with BMI. Analysis of the time-based components (P1 latency, $\mathrm{p}=0.819 ; \mathrm{P} 1$ duration, $\mathrm{p}=0.515$ ) indicated that the larger acceleration magnitudes in the patients with increased BMI were not associated with a related change in temporal parameters. Adjusting for gender strengthened the relationship between BMI and P1 amplitude $(r=-0.490, p=0.005)$ when gender was factored into the analysis ( $\mathrm{p}$-value reduced from 0.023 to 0.005 ).

\section{Age}

Age had no significant relationship with any of the SMT components (P1 amplitude, $\mathrm{p}=0.693 ; \mathrm{P} 1$ latency, $\mathrm{p}=0.131 ; \mathrm{P} 1$ duration, $\mathrm{P}=0.329 ; \mathrm{P} 1 \_\mathrm{AUC} \mathrm{p}=0.408$ ). Gender did not influence this partial correlation $(\mathrm{p}>0.69)$. 


\section{Discussion}

The exact nature of the thrust delivered during spinal manipulative therapy is not well understood. Although spinal manipulation is often described as a high velocity and low amplitude maneuver, these parameters have never been directly quantified. To date, while force-time histories have been reported with respect to the manipulative input, the kinematic nature of the thrust phase of SMT has not been characterized. The primary objective of this study was to address this issue by using accelerometers to study the hand kinematics produced during manipulation of the cervical, thoracic, and lumbar spine as well as the sacroiliac (SI) joint. As such, the present study represents the first report of the acceleration properties of the hand movements that are used to generate the thrust phase of SMT. Notable differences were observed in the magnitudes of the accelerations across different spinal levels. Acceleration magnitudes were not influenced by gender at the cervical or thoracic levels but larger amplitudes (P1 amplitude, P1P2 amplitude, P1_AUC) were seen in males at the lumbar and SI levels. These large differences in acceleration amplitude were not accompanied by similar changes in temporal parameters either across or within levels of the spinal column. Further, gender had little or no impact on temporal parameters and their relationship with height, body mass, BMI and age. 
As this is the first study to measure hand acceleration during SMT there are no comparative data available. However, an approximation can be made to the data reported by Herzog and co-workers who used accelerometers attached to the skin over the thoracic spine to measure the acceleration that occurred in response to manipulation (Herzog et al., 1993a; Herzog et al., 1997). Overall, the acceleration-time histories presented here are similar to those of Herzog and co-workers who reported the tri-phasic signal was 100-200 ms in duration. In addition, by comparing normalized force-time and acceleration-time histories, Herzog was able to determine that peak force corresponds approximately to the peak of the P2 phase of acceleration. The thoracic manipulation studies conducted by Herzog (1993a, 1997 and 2001) reported a time to peak force of 120-130 ms analogous to the $150 \mathrm{~ms}$ of the P2 latency from the present study.

Although temporal parameters were similar across spinal levels, clear differences in the magnitude of acceleration were seen between anatomical regions. In particular, lumbar manipulation had the largest $\mathrm{P} 1$ peak amplitude $\left(17.9 \mathrm{~m} / \mathrm{s}^{2}\right)$ followed by cervical manipulation $\left(8.9 \mathrm{~m} / \mathrm{s}^{2}\right)$. The lowest amplitudes of the $\mathrm{P} 1$ phase were seen during thoracic and sacroiliac manipulations with negligible differences in P1 peak amplitude between these levels (thoracic: $2.80 \mathrm{~m} / \mathrm{s}^{2}$, SI: $4.40 \mathrm{~m} / \mathrm{s}^{2}$ ). The same pattern was found between spinal levels for P2 amplitude.

The differences in acceleration across the spinal levels were not unexpected given that similar results were reported for force-time data (Herzog et al., 1993a). Surprisingly, the specific reasons for these differences have not been well elaborated. It has been assumed that the nature of the resistance of the spinal tissues to movement, as determined 
by the clinician's palpation and during the preload phase, is a primary determinant of the magnitude of the manipulative thrust (Harms et al., 1999; Herzog et al., 1993b). From an anatomical perspective, the resistance of the spinal tissues to passive movement would be expected to gradually increase from the cervical through to the SI regions. Accordingly, it would also be expected that thrust amplitudes, and the accelerations required to generate those thrusts, would increase from cervical to lumbar with the SI joint requiring the largest SMT amplitudes. Indeed, this increasing pattern from cervical to SI was seen when the manipulative forces were measured (Herzog et al., 1993a; Herzog et al., 1993b). Specifically, the peak forces were considerably smaller for SMT delivered to the cervical spine compared to those for the thoracic and SI levels. This gradation is consistent with anecdotal reports from clinicians that smaller thrusts are delivered to the cervical spine.

Therefore, it was unexpected that acceleration magnitudes during cervical spine manipulation were greater than those measured during thoracic and SI manipulation. The reason for this is unclear especially considering the reported forces (Herzog et al., 1993a; Herzog et al., 1993b) and a lack of intent by clinicians to deliver a large thrust at the cervical level. It is possible that a methodological issue may factor into the force differences between cervical and thoracic manipulations. The typical device used in these studies to measure forces sums the pressure inputs across the entire contact area. Therefore, a manipulation with a larger contact area has the potential to generate larger total forces. The contact area of the clinician's hand during cervical spine manipulation is much smaller than the contact area of the clinician's hand during thoracic manipulation. 
Further, the contact area of a thoracic manipulation increases during the thrust phase and this nonspecific contact contributes considerably to the total force (Herzog et al., 2001). It is unknown whether the same differences between cervical and thoracic manipulations would be observed if the total forces were adjusted for the relatively small specific contact area (e.g., transverse process). The issue of contact area does not impact directly on acceleration measurements and, therefore, would not account for the higher acceleration magnitudes observed in the present study for cervical versus thoracic manipulations.

An alternate explanation for the differences between cervical and thoracic manipulations arises from the anatomical nature of the cervical spine and the specific SMT technique utilized. The cervical manipulation utilized in this study was a rotary technique which may have been more effective at elongating and tensing the connective tissues at the cervical level compared to other spinal levels. This in turn may have resulted in a tighter coupling between the clinician's hand and the target tissues and produced greater accelerations due to the reduced damping of the cervical connective tissues. In addition, the relatively smaller scale of the tissues overlying the cervical spine would reduce any damping from these components. Together, these factors may combine to increase the acceleration measured at the cervical spine despite the application of a lower manipulative force. Future studies would need to combine acceleration and force measurements to confirm this mechanism. Additionally, the above methodological issues regarding force measurement would need to be considered in such a study.

The largest acceleration waveforms were observed during manipulation of the 
lumbar spine. Since the goal of spinal manipulation is to produce a relative movement of a spinal segment, the larger scale and strength of the anatomic structures of the lumbar spine would suggest a larger manipulative input would be required compared to other spinal levels. Clinically, the generation of larger thrusts is achieved by incorporating the clinician's body mass in the development of the preload and manipulative thrust. This is distinct from other regions where most, or all, of the thrust is developed through the arms with the trunk primarily acting as a stabilizing component. In the present study lumbar manipulations were performed with the patient in a side-lying position which permits use of the clinician's body mass in the delivery of the thrust, a critical aspect of the efficacy of this manipulation (Peterson \& Bergmann, 2002). In contrast, the SI manipulation, which is also performed with the patient in side-lying, does not permit effective reinforcement of the thrust with the clinician's body mass due to the need to apply the thrust in a horizontal plane. In practice, the SI thrusts rely to a great extent on the application of a lateral input from the upper extremity (Peterson \& Bergmann, 2002; Downie et al., 2010). The inability to transfer this body mass component likely accounts for the lower accelerations seen in the SI manipulations relative to those at the lumbar level. Further, since the SI thrust is generated with a single arm, the thrusting shoulder must also attempt to stabilize the action which may account for the distinct off-axis motion that gives rise to the bi-phasic peak that occurred during the P1 phase of SI manipulation.

The temporal aspects of manipulation proved to be fairly similar between spinal levels. The shortest times to P1 peak were observed during lumbar (49 ms) and cervical 
(58 ms) SMT, followed closely by thoracic $(71 \mathrm{~ms})$ manipulation. A longer P1 latency was observed for sacroiliac (132 ms) manipulation. Although these temporal parameters cannot be directly compared to those reported for SMT forces, the time to peak force is roughly equivalent to time to P2 peak (Herzog et al., 1997). The actual mean values for time to peak force established by Herzog's group was $48 \mathrm{~ms}$ for cervical manipulation and $150 \mathrm{~ms}$ for thoracic and SI manipulation. By comparison peak P2 latencies in our study were as follows: cervical (132 ms), thoracic (147 ms), lumbar (92 ms) and sacroiliac (205 ms).

Overall, the manipulations had a very similar time-base except for those applied to the SI joint which were longer in duration. This is in contrast to the large differences in amplitude between manipulations at the different spinal levels. Similarly, treatment forces across clinicians can vary by a factor of 10 and yet the time to peak thrust is fairly constant (120-130 ms in the case of thoracic manipulation) (Herzog, 2000). That the acceleration amplitudes showed little relationship with acceleration latencies suggests SMT thrusts of differing amplitudes are not generated in a scaling manner whereby a larger magnitude manipulation is necessarily associated with a longer latency. This would support accepting the main hypothesis of the present study that the primary variable of SMT is the thrust amplitude whereas the temporal aspect is relatively stable. A plausible explanation for this finding is that the clinician's intent for the magnitude of the manipulative thrust is based on the level of the spine being treated. That is, due consideration is given to the unique anatomy of the spinal region including joint orientation, connective tissue and disc thickness, and muscle bulk (Herzog et al., 1993b). 
These factors appear to contribute to the clinical decision of how much thrust input is to be delivered independent of the rate at which the thrust will be applied, which seems relatively constant.

Spinal manipulative therapy has been described as a high velocity and low amplitude thrust, yet neither characteristic has been quantified experimentally. The present study measured acceleration and calculated velocity through numerical integration of acceleration. While the velocity values are included here for reference (along with the displacement values for thoracic SMT), there is no known comparative values for other manual therapies such as mobilization. Although the present data add some quantitative aspect to the high velocity, low amplitude description of SMT, these data contribute far more to the potential to characterize all SMT techniques according to acceleration parameters. Acceleration proved highly effective in generating distinctive waveforms and amplitude-latency patterns between the four SMT techniques. Thus, acceleration may be suitable to categorize various SMT techniques according to these properties.

To further examine the potential relationships between the SMT amplitude and temporal parameters at different spinal levels, separate analyses were conducted between P1 amplitude and P1 latency, which determined that these two parameters are not correlated with one another. Additionally, P1 amplitude and P1 latency were tested for correlations with the other amplitude and temporal parameters of acceleration. In general, P1 amplitude was significantly correlated with other amplitude factors and P1 latency was correlated with other temporal parameters. Notable was the lack of correlations 
between amplitude and temporal aspects of the SMT acceleration waveforms. This is consistent with similar findings from SMT force studies which reported that manipulations had a relatively similar time-base even though the SMT forces differed at each spinal level (Herzog, 2000). These findings suggest that the magnitude of the thrust is derived independently of temporal considerations. Presumably, the desire to achieve a high velocity thrust translates into relatively fixed time base for SMT with the amplitude of the thrust being the variable that is primarily adjusted to each clinical situation.

A further investigation, beyond the relationships between amplitude and temporal parameters, explored whether specific features that occurred early in the manipulation were more or less coupled to later events within the acceleration waveform. The rationale for this was based on a simplistic 2 component model of SMT whereby the acceleration measured from the hand was a function of 1) the input from the clinician and 2) the resistance of the patient's tissues to that input. If such a relationship existed, it was assumed that P1 and P2 would be most representative of the clinician's input and the tissue resistance to that input, respectively. It follows then, that the earliest portions of P1 would be least influenced by the response of the tissue to the input. Two parameters were derived that attempted to capture the clinician's intent; the slope of the rising phase of P1 and the peak jerk that occurred during the rising phase of P1. Correlation analysis revealed a separate pattern for cervical, thoracic and lumbar regions.

As expected, the slope and peak jerk of the rising P1 phase were highly correlated to $\mathrm{P} 1$ peak amplitude with $\mathrm{r}$ values ranging from $0.77-0.91$. Interestingly, the correlations between these early events and the P2 peak differed across spinal levels. At 
the thoracic level the correlation between the early parameters and the P2 peak reached the same $r$ values as those for the P1 peak. At the cervical level the correlations were only half as strong, although still statistically significant. In contrast, there were no significant correlations between the $\mathrm{P} 2$ peaks and these early events at the lumbar level. Taken at face value, these highly preliminary findings suggest that little or no distinction can be made between early and later components of the acceleration waveform for thoracic and cervical manipulations. This may reflect a high degree of coupling between the clinician's hand and the tissues at these levels such that the acceleration waveform has a high degree if internal correlation. In contrast, there appears to be separate factors that contribute to P1 and P2 at the lumbar level. It may be that the thicker overlying tissues make it difficult to achieve the same effective coupling during the preload phase and that the clinician's hand undergoes an initial phase of thrust during which additional soft tissue compression or elongation occurs. This additional process during the lumbar thrust phase may account for the lack of correlation between the early P1 events and the peak P2 acceleration. Ultimately, a full understanding of the biological significance of the various components of the acceleration waveform would require simultaneous collection of several outcome measures including acceleration and force data combined with detailed image analysis as a minimum.

The secondary objective of this study was to determine whether the anthropometric characteristics of the patients receiving SMT correlate with the kinematic parameters of SMT. It has already been suggested that patient age and body size do influence the magnitude of the clinician's manipulative input (Kirstukas \& Backman, 
1999; Harms et al., 1999). This study sought to determine the extent to which height, body mass, body mass index (BMI) and age could account for differences in SMT characteristics seen between individuals for each level of spinal manipulation. Additional analysis utilizing partial correlations was conducted to control for gender and its potential influence on the relationship between the patient's anthropometric characteristics and P1 amplitude.

At the cervical level, height and body mass had little relationship with acceleration amplitudes although longer SMT latencies were associated with taller or heavier patients. In contrast, greater acceleration magnitudes were seen in those who were older or those with a higher BMI. These same factors had little relationship to changes in temporal parameters. While it seems sensible that a higher BMI would be associated with a greater thrust input, the observation of greater accelerations in older patients was not expected. Interestingly, while both BMI and age were positively correlated with acceleration amplitudes, there was an inverse relationship between BMI and age indicating that age is an independent predictor of higher SMT amplitudes. Partial correlations were significant for the height-P1amplitude when controlling for gender indicating that taller male subjects received larger amplitude cervical manipulations. Adjusting for gender did not influence the outcome of correlations between body mass, BMI and age with cervical P1 amplitude.

In a related study, a larger thrust force was recorded in an older age group during grade I and II mobilizations of the lumbar spine in comparison to a younger group (Harms et al., 1999). These authors suggested that a change in the soft tissue compliance 
of the spine necessitated the application of a greater force to achieve a similar degree of displacement of the spinal segment. The changes in tissue compliance would also be expected to contribute to greater acceleration amplitudes from the same level of thrust force if these changes reduced soft tissue damping of the thrust. Therefore, older patients in the present study may not have received a larger amplitude thrust. Rather, the altered viscoelastic properties of the cervical tissue may have created larger accelerations from a relatively low thrust force.

With respect to thoracic manipulation, there were no significant relationships between patient anthropometric characteristics or age in comparison with the magnitude and temporal parameters of acceleration. Similarly, no significant correlations were present between anthropometric parameters and lumbar SMT characteristics. The one exception was between height and lumbar P2 peak amplitude $\left(\mathrm{r}^{2}=0.25\right)$; no similar relationship was present for height and P1 amplitude. While consistent with the above mentioned dissociation of P1 and P2 amplitudes at the lumbar level, it must also be recognized that this isolated finding may be due to a type I error.

By comparison, a study conducted by Harms et al (1999), examined the association between the physical characteristics of their subjects, including range of motion, age, weight and height, with the maximum forces used during graded mobilization of the lumbar spine. They also noted a lack of consistency in the association between the physical characteristics of the subject and the force applied to the spine. Specifically, weight and height could not account for the variation in the magnitude of 
the mobilization force to the lumbar spine.

Somewhat surprisingly, body mass and BMI were consistently associated with SMT magnitude for SI manipulations only, highlighting the relatively weak association between the anthropometric features and the acceleration parameters. Controlling for gender had little effect overall on these correlations. This would indicate that additional factors are involved in the process of determining the magnitude of the manipulative thrust in the clinical setting. Within the confines of the present study, the hypothesis that the anthropometric characteristics of the patients receiving SMT correlate with the kinematic parameters of SMT would have to be rejected. This issue will require additional studies that are specifically designed to assess these relationships including a larger number of anthropometric factors and a larger sample size.

\section{Limitations}

The patient group used for this study was a convenience sample from the principal investigator's chiropractic clinic. Recruiting the same sample size from a larger number of clinics would provide for greater generalization of the results. However, the correlations between anthropometric and SMT parameters would have been less robust if the same sample size was drawn from several clinics rather than one assuming the same 
high level of variability in acceleration data as that for force data when comparing across clinicians (Herzog 1993a). Comparing acceleration data across clinicians would also address the inherent bias created in this study whereby the principal investigator was also the clinician performing the manipulations. However, it has been noted by Herzog that the peak force corresponds approximately to the peak of the P2 phase of acceleration. The thoracic manipulations conducted by Herzog (1993a; 1997 and 2001) report a time to peak force of $120-130 \mathrm{~ms}$ analogous to the $150 \mathrm{~ms}$ of the P2 latency of the present study. The latency data from the present study are at least consistent with that reported elsewhere and argue against the presence of bias. Further, this was an observational study rather than an intervention study which reduces potential motivation for bias.

Preload was not measured in the present study. Herzog et al (1993a; 1993b) reported that variations in preload force are an important factor in determining peak thrust force. At the same time, the change in force from preload to peak force (thrust force) was the factor that most strongly predicted peak force. Given this, and that no previous study had assessed hand kinematics during SMT, it was decided to focus on the acceleration aspect of SMT. Combining force and acceleration measurements in the same study would provide further insight into the SMT process.

Peak displacement values were estimated from the thoracic acceleration data only. These manipulations were performed with a linear application of the thrust along a relatively pure posterior-anterior axis combined with a return of the thrust hand to the same position from which it started, resulting in a clear P3 phase of the acceleration waveform. No other manipulation met these requirements to permit reasonable estimates 
of displacement using numerical integration of the acceleration data. Although no comparison values are known to exist, the thoracic displacement values are included here for reference.

Power analysis was done a priori based on our primary objective of determining the acceleration characteristics of SMT and whether these characteristics vary according to the level of the spine at which SMT is performed. As a result, this study was not deliberately powered to address the second objective; if the anthropometric parameters of patients receiving SMT correlate with the kinematic parameters of SMT. Post-hoc power calculations for the correlational analyses determined that this aspect of the analysis was considerably under-powered in that a sample size approximately double that used would have been needed. Therefore, the non-significant outcomes in the present study for the associations between the anthropometric characteristics of the patient with the kinematic parameters of the SMT may reflect type II error.

\section{Conclusions}

This is the first study to assess the characteristics of spinal manipulative therapy performed in a clinical population. The acceleration data revealed clear differences in the magnitude of accelerations generated between different levels of the spine. In contrast, 
relatively small differences were present in the temporal parameters between the spinal levels. Overall, anthropometric factors showed relatively weak associations with acceleration parameters suggesting that additional factors are involved in the process of determining the magnitude of the SMT thrust in the clinical setting.

This study also serves to illustrate the usefulness of applying accelerometers in the investigation of SMT. Accelerometers offer one means of categorizing different spinal manipulative techniques. In addition, it may be possible to reverse-engineer various sham manipulations to explore the mechanisms of SMT by deliberately modifying amplitude or temporal aspects of the SMT acceleration waveform. Finally, accelerometers could be used as an evaluation tool for instructing students in spinal manipulation by providing a model of a characteristic acceleration profile for them to reproduce at the different regions of the spinal column.

\section{Reference List}

Assendelft, W. J., Morton, S. C., Yu, E. I., Suttorp, M. J., \& Shekelle, P. G. (2003). Spinal manipulative therapy for low back pain. A meta-analysis of effectiveness relative to other therapies. Annals of Internal Medicine, 138, 871-881. 
Assendelft, W. J., Morton, S. C., Yu, E. I., Suttorp, M. J., \& Shekelle, P. G. (2004). Spinal manipulative therapy for low back pain. Cochrane.Database.Syst.Rev., CD000447.

Cherkin, D. C., Deyo, R. A., Battie, M., Street, J., \& Barlow, W. (1998). A comparison of physical therapy, chiropractic manipulation, and provision of an educational booklet for the treatment of patients with low back pain. New England Journal of Medicine, 339, 1021-1029.

Colloca, C. J. \& Keller, T. S. (2004). Active trunk extensor contributions to dynamic posteroanterior lumbar spinal stiffness. J.Manipulative Physiol Ther., 27, 229237.

Colloca, C. J., Keller, T. S., \& Gunzburg, R. (2003). Neuromechanical characterization of in vivo lumbar spinal manipulation. Part II. Neurophysiological response. J.Manipulative Physiol Ther., 26, 579-591.

Colloca, C. J., Keller, T. S., \& Gunzburg, R. (2004). Biomechanical and neurophysiological responses to spinal manipulation in patients with lumbar radiculopathy. J.Manipulative Physiol Ther., 27, 1-15.

Colloca, C. J., Keller, T. S., Harrison, D. E., Moore, R. J., Gunzburg, R., \& Harrison, D. D. (2006). Spinal manipulation force and duration affect vertebral movement and neuromuscular responses. Clin.Biomech.(Bristol., Avon.), 21, 254-262.

Colloca, C. J., Keller, T. S., Moore, R. J., Gunzburg, R., \& Harrison, D. E. (2007). 
Intervertebral disc degeneration reduces vertebral motion responses. Spine, 32, E544-E550.

Cramer, G., Budgell, B., Henderson, C., Khalsa, P., \& Pickar, J. (2006). Basic science research related to chiropractic spinal adjusting: the state of the art and recommendations revisited. J.Manipulative Physiol Ther., 29, 726-761.

Dishman, J. D., Dougherty, P. E., \& Burke, J. R. (2005). Evaluation of the effect of postural perturbation on motoneuronal activity following various methods of lumbar spinal manipulation. Spine J., 5, 650-659.

Downie, A. S., Vemulpad, S., \& Bull, P. W. (2010). Quantifying the high-velocity, lowamplitude spinal manipulative thrust: a systematic review. J.Manipulative Physiol Ther., 33, 542-553.

Fernandez-de-las-Penas, C., Palomeque-del-Cerro, L., Rodriguez-Blanco, C., GomezConesa, A., \& Miangolarra-Page, J. C. (2007). Changes in neck pain and active range of motion after a single thoracic spine manipulation in subjects presenting with mechanical neck pain: a case series. J.Manipulative Physiol Ther., 30, 312320.

Gal, J., Herzog, W., Kawchuk, G., Conway, P., \& Zhang, Y. T. (1997a). Measurements of vertebral translations using bone pins, surface markers and accelerometers. Clin.Biomech.(Bristol., Avon.), 12, 337-340.

Gal, J., Herzog, W., Kawchuk, G., Conway, P. J., \& Zhang, Y. T. (1997b). Movements of 
vertebrae during manipulative thrusts to unembalmed human cadavers. J.Manipulative Physiol Ther., 20, 30-40.

Gal, J. M., Herzog, W., Kawchuk, G. N., Conway, P. J., \& Zhang, Y. T. (1995). Forces and relative vertebral movements during SMT to unembalmed post-rigor human cadavers: peculiarities associated with joint cavitation. J.Manipulative Physiol Ther., 18, 4-9.

Haas, M. (1990a). The physics of spinal manipulation. Part I. The myth of $F=$ ma. J.Manipulative Physiol Ther., 13, 204-206.

Haas, M. (1990b). The physics of spinal manipulation. Part II. A theoretical consideration of the adjustive force. J.Manipulative Physiol Ther., 13, 253-256.

Haas, M. (1990c). The physics of spinal manipulation. Part III. Some characteristics of adjusting that facilitate joint distraction. J.Manipulative Physiol Ther., 13, 305308.

Harms, M. C., Innes, S. M., \& Bader, D. L. (1999). Forces measured during spinal manipulative procedures in two age groups. Rheumatology.(Oxford), 38, 267-274.

Herzog, W. (2000). The Mechanical, Neuromuscular, and Physiologic Effects Produced by Spinal Manipulation. In Clinical Biomechanics of Spinal Manipulation (First Edition ed., pp. 191-207). Philadelphia: Churchill Livingstone.

Herzog, W., Conway, P. J., Kawchuk, G. N., Zhang, Y., \& Hasler, E. M. (1993a). Forces 
exerted during spinal manipulative therapy. Spine, 18, 1206-1212.

Herzog, W., Kats, M., \& Symons, B. (2001). The effective forces transmitted by highspeed, low-amplitude thoracic manipulation. Spine, 26, 2105-2110.

Herzog, W., Kawchuk, G., \& Conway, P. (1993b). Relationship Between Preload and Peak Forces During Spinal Manipulative Treatments. Journal of the Neuromusculoskeletal System, 1, 52-58.

Herzog, W., Suter, E., \& Conway, P. J. (1997). Accelerations recorded from the spinous processes during spinal manipulative treatments of the thoracic spine. Australas.Chiropr.Osteopathy., 6, 75-79.

Hurwitz, E. L., Morgenstern, H., Harber, P., Kominski, G. F., Yu, F., \& Adams, A. H. (2002). A randomized trial of chiropractic manipulation and mobilization for patients with neck pain: clinical outcomes from the UCLA neck-pain study. American Journal of Public Health, 92, 1634-1641.

Ianuzzi, A. \& Khalsa, P. S. (2005a). Comparison of human lumbar facet joint capsule strains during simulated high-velocity, low-amplitude spinal manipulation versus physiological motions. Spine J., 5, 277-290.

Ianuzzi, A. \& Khalsa, P. S. (2005b). High loading rate during spinal manipulation produces unique facet joint capsule strain patterns compared with axial rotations. J.Manipulative Physiol Ther., 28, 673-687. 
Kawchuk, G. N., Herzog, W., \& Hasler, E. M. (1992). Forces generated during spinal manipulative therapy of the cervical spine: a pilot study. J.Manipulative Physiol Ther., 15, 275-278.

Kawchuk, G. N. \& Perle, S. M. (2009). The relation between the application angle of spinal manipulative therapy (SMT) and resultant vertebral accelerations in an in situ porcine model. Man.Ther., 14, 480-483.

Kawchuk, G. N., Prasad, N. G., McLeod, R. C., Liddle, T., Li, T., \& Zhu, Q. (2006). Variability of force magnitude and force duration in manual and instrument-based manipulation techniques. J.Manipulative Physiol Ther., 29, 611-618.

Keller, T. S. \& Colloca, C. J. (2002). A rigid body model of the dynamic posteroanterior motion response of the human lumbar spine. J.Manipulative Physiol Ther., 25, 485-496.

Keller, T. S., Colloca, C. J., \& Gunzburg, R. (2003). Neuromechanical characterization of in vivo lumbar spinal manipulation. Part I. Vertebral motion. J.Manipulative Physiol Ther., 26, 567-578.

Keller, T. S., Colloca, C. J., Moore, R. J., Gunzburg, R., \& Harrison, D. E. (2006a). Increased multiaxial lumbar motion responses during multiple-impulse mechanical force manually assisted spinal manipulation. Chiropr.Osteopat., 14, 6.

Keller, T. S., Colloca, C. J., Moore, R. J., Gunzburg, R., Harrison, D. E., \& Harrison, D. D. (2006b). Three-dimensional vertebral motions produced by mechanical force 
spinal manipulation. J.Manipulative Physiol Ther., 29, 425-436.

Kirstukas, S. J. \& Backman, J. A. (1999). Physician-applied contact pressure and table force response during unilateral thoracic manipulation. J.Manipulative Physiol Ther., 22, 269-279.

Koes, B. W., Assendelft, W. J., van der Heijden, G. J., \& Bouter, L. M. (1996). Spinal manipulation for low back pain. An updated systematic review of randomized clinical trials. Spine, 21, 2860-2871.

Lee, R. Y., McGregor, A. H., Bull, A. M., \& Wragg, P. (2005). Dynamic response of the cervical spine to posteroanterior mobilisation. Clin.Biomech.(Bristol., Avon.), 20, $228-231$.

Lehman, G. J. \& McGill, S. M. (2001). Spinal manipulation causes variable spine kinematic and trunk muscle electromyographic responses. Clin.Biomech.(Bristol., Avon.), 16, 293-299.

Lehman, G. J., Vernon, H., \& McGill, S. M. (2001). Effects of a mechanical pain stimulus on erector spinae activity before and after a spinal manipulation in patients with back pain: a preliminary investigation. J.Manipulative Physiol Ther., 24, 402-406.

Maigne, J. Y. \& Guillon, F. (2000). Highlighting of intervertebral movements and variations of intradiskal pressure during lumbar spine manipulation: a feasibility study. J.Manipulative Physiol Ther., 23, 531-535. 
Meade, T. W., Dyer, S., Browne, W., Townsend, J., \& Frank, A. O. (1990). Low back pain of mechanical origin: randomised comparison of chiropractic and hospital outpatient treatment. BMJ, 300, 1431-1437.

Peterson, D. H. \& Bergmann, T. F. (2002). The Spine: Anatomy, Biomechanics, Assessment, and Adjustive Techniques. In Chiropractic Technique (Second Edition ed., pp. 175-339). Mosby, Inc.

Pickar, J. G. \& Kang, Y. M. (2006). Paraspinal muscle spindle responses to the duration of a spinal manipulation under force control. J.Manipulative Physiol Ther., 29, $22-31$

Pickar, J. G., Sung, P. S., Kang, Y. M., \& Ge, W. (2007). Response of lumbar paraspinal muscles spindles is greater to spinal manipulative loading compared with slower loading under length control. Spine J., 7, 583-595.

Rogers, C. M. \& Triano, J. J. (2003). Biomechanical measure validation for spinal manipulation in clinical settings. J.Manipulative Physiol Ther., 26, 539-548.

Ross, J. K., Bereznick, D. E., \& McGill, S. M. (2004). Determining cavitation location during lumbar and thoracic spinal manipulation: is spinal manipulation accurate and specific? Spine, 29, 1452-1457.

Shekelle, P. G., Adams, A. H., Chassin, M. R., Hurwitz, E. L., \& Brook, R. H. (1992). Spinal manipulation for low-back pain. Annals of Internal Medicine, 117, 590598. 
Solinger, A. B. (2000). Theory of small vertebral motions: an analytical model compared to data. Clin.Biomech.(Bristol., Avon.), 15, 87-94.

Sran, M. M., Khan, K. M., Zhu, Q., McKay, H. A., \& Oxland, T. R. (2004). Failure characteristics of the thoracic spine with a posteroanterior load: investigating the safety of spinal mobilization. Spine, 29, 2382-2388.

Sung, P. S., Kang, Y. M., \& Pickar, J. G. (2005). Effect of spinal manipulation duration on low threshold mechanoreceptors in lumbar paraspinal muscles: a preliminary report. Spine, 30, 115-122.

Symons, B. P., Leonard, T., \& Herzog, W. (2002). Internal forces sustained by the vertebral artery during spinal manipulative therapy. J.Manipulative Physiol Ther., $25,504-510$.

Triano, J. J. (2001). Biomechanics of spinal manipulative therapy. Spine J., 1, 121-130.

Tsung, B. Y., Evans, J., Tong, P., \& Lee, R. Y. (2005). Measurement of lumbar spine loads and motions during rotational mobilization. J.Manipulative Physiol Ther., $28,238-244$.

Webber, S. C. \& Kriellaars, D. J. (2004). The effect of stabilization instruction on lumbar acceleration. Clin.Biomech.(Bristol., Avon.), 19, 777-783.

\section{Appendix A}


Research Participant Information and Consent Form (Patient)

Title of Study: Acceleration of clinician hand movements during spinal manipulative therapy

Principal Investigator: Geoff Gelley DC, FRCCSS(C)

12-845 Dakota St.

Winnipeg, MB R2M 5M3

(204)254-0130

You are being asked to participate in a research study. Please take your time to review the consent form and discuss any questions you may have with the study staff. You may take your time to make your decision about participating in this study and you may discuss it with your friends, family or (if applicable) your doctor before you make your decision. This consent form may contain words that you do not understand. Please ask the study staff to explain any words or information that you do not clearly understand.

\section{Purpose of Study}

The purpose of this research study is to measure the hand movements of a chiropractor during spinal manipulative therapy (SMT). In addition we wish to determine if these hand movements change according to the physical characteristics of the person receiving SMT.

A total of 60-80 patients will participate in this study in addition to 2-3 chiropractic clinicians. 


\section{Study procedures}

All patients are being recruited for this study from chiropractic clinics in the City of Winnipeg. Your chiropractor has participated in a research study to describe the physical movements they generate during the delivery of SMT. We are conducting a separate study to determine if these physical movements are dependent on various aspects of those receiving SMT, for example, age or height of the patient. If you consent, one of the clinic staff members will obtain your date of birth, gender, height and body mass. It is expected that these procedures will lengthen your appointment by 10-15 minutes on this one occasion. No other requirements are needed for this study.

You can stop participating at any time. However, if you decide to stop participating in this study, we encourage you to talk to the study staff first.

\section{Risks and Discomforts}

No additional risks or discomfort are expected to occur as a result of your participation in this study.

\section{Benefits}

There may or may not be direct benefit to you from participating in this study. We hope the information learned from this study will benefit researchers studying spinal manipulation or students in educational institutions that instruct spinal manipulation. 


\section{Costs}

All the procedures that will be performed as part of this study are provided at no additional cost to you outside of your chiropractor's usual billing fee.

\section{Payment for participation}

You will receive no payment or reimbursement for any expenses related to taking part in this study.

\section{Confidentiality}

Information gathered in this research study may be published or presented in public forums; however your name and other identifying information will not be used or revealed. Despite efforts to keep your personal information confidential, absolute confidentiality cannot be guaranteed. Your personal information may be disclosed if required by law. All data files including those with personal information will bear only your assigned study number. A separate document will contain the master list of only names and study numbers.

Organizations that may inspect and/or copy your research records for quality assurance and data analysis include groups such as: The Faculty of Graduate Studies at the University of Manitoba and The Master of Science in Medical Rehabilitation Department at The School of Medical Rehabilitation of The University of Manitoba. 
The University of Manitoba Human Research Ethics Board may review records related to the study for quality assurance purposes.

All records will be kept in a locked secure area and only those persons identified will have access to these records If any of your research records need to be copied to any of the above, your name and all identifying information will be removed. No information revealing any personal information such as your name, address or telephone number will leave The University of Manitoba.

\section{Voluntary Participation/Withdrawal from the Study}

Your decision to take part in this study is voluntary. You may refuse to participate or you may withdraw from the study at any time. Your decision not to participate or withdraw from the study will not affect your care at this clinic. If the study staff feels that it is in your best interest to withdraw you from the study, they will remove you without your consent.

We will tell you about any new information that may affect your health, welfare or willingness to stay in the study.

\section{Health Care for Injury Related to the Study}

In the case of injury resulting from this study, necessary treatment will be available at no 
additional cost to you.

You are not waiving any of your legal rights by signing this consent form or releasing the investigator(s) or the sponsors(s) from their legal and professional responsibilities.

\section{Questions}

You are free to ask any questions that you may have about your treatment and your rights as a research participant. If any questions come up during or after the study or if you have a research-related injury, contact the study doctor: Geoff Gelley at (204)2540130.

For questions about your rights as a research participant, you may contact The University of Manitoba, Bannatyne Campus Research Ethics Board Office at (204)7893389.

Do not sign this consent form unless you have had a chance to ask questions and have received satisfactory answers to all of your questions. 


\section{Statement of Consent}

I have read this consent form. I have had the opportunity to discuss this research study with the research study staff. I have had my questions answered by them in language I understand. The risks and benefits have been explained to me. I believe that I have not been unduly influenced by any study team member to participate in the research study by any statements or implied statements. Any relationship (such as employer, supervisor or family member) I have with the study team has not affected my decision to participate. I understand that I will be given a copy of this consent form after signing it. I understand that my participation in this study is voluntary and that I may choose to withdraw at any time. I freely agree to participate in this research study.

I understand that information regarding my personal identity will be kept confidential, but that confidentiality is not guaranteed. I authorize the inspection of any of my records that relate to this study by The University of Manitoba Research Ethics Board for quality assurance purposes.

By signing this consent form, I have not waived any legal rights that I have as a participant in a research study.

I agree to be contacted for future follow-up in relation to this study: Yes_ No_ 
Participant signature Date (Day/month/year)

Participant printed name: 


\section{Research Participant Information and Consent Form (Chiropractors)}

Title of Study: Acceleration of clinician hand movements during spinal manipulative therapy

Principal Investigator: Geoff Gelley DC, FRCCSS(C)

12-845 Dakota St.

Winnipeg, MB R2M 5M3

(204)254-0130

You are being asked to participate in a research study. Please take your time to review the consent form and discuss any questions you may have with the study staff. You may take your time to make your decision about participating in this study and you may discuss it with your friends, family or (if applicable) your doctor before you make your decision. This consent form may contain words that you do not understand. Please ask the study staff to explain any words or information that you do not clearly understand.

\section{Purpose of Study}

This research study is being conducted to quantify spinal manipulative therapy (SMT) utilizing a triaxial accelerometer. Specifically, we wish to characterize the specific hand accelerations of spinal manipulation performed at different segments of the spine. In addition we wish to determine if acceleration profiles are correlated with physical characteristics of those receiving SMT. 
A total of 2-3 chiropractic clinicians will participate in this study.

\section{Study procedures}

All chiropractors are being recruited for this study from chiropractic clinics in the City of Winnipeg. A third party will be present to record findings and attend to the computer

and instrumentation. You will assess your patients for spinal joint dysfunction as per the study protocol. You will also be weighed and your height will be measured.

You will provide spinal manipulation to the involved areas of your patient's spine as you would have done at any previous treatment sessions. The treatment sessions will be scheduled during a convenient time at your chiropractic clinic. You will place your patient in the appropriate position and perform the appropriate manipulation to a specific spinal joint. Prior to performing the manipulation, a triaxial accelerometer will be attached to the back of your manipulating hand with adhesive tape. The accelerometer data will be collected through wireless transmission to a portable computer. Following your manipulations you will complete a questionnaire pertaining to the quality of your manipulations. The collection of this information is expected to require an additional 1015 minutes during the treatment session.

You can stop participating at any time. However, if you decide to stop participating in this study, we encourage you to talk to the study staff first. 
Approximately six months following your participation in the study, aggregate results will be provided to you for your interest.

\section{Risks and Discomforts}

Possible skin irritation may occur from the adhesive tape fixing the accelerometer to the back of your hand. No other risks or discomforts are expected to occur while you have the accelerometer attached to your hand when you perform spinal manipulation.

\section{Benefits}

There may or may not be direct benefit to you from participating in this study. We hope the information learned from this study will benefit researchers studying spinal manipulation or students in educational institutions that instruct spinal manipulation.

\section{Costs}

All the procedures that will be performed as part of this study are provided at no additional cost to you.

\section{Payment for participation}

You will receive no payment or reimbursement for any expenses related to taking part in this study outside of your usual billing fees to patients or third parties.

\section{Confidentiality}


Information gathered in this research study may be published or presented in public forums; however your name and other identifying information will not be used or revealed. Despite efforts to keep your personal information confidential, absolute confidentiality cannot be guaranteed. Your personal information may be disclosed if required by law. All data files including those with personal information will bear only your assigned study number. A separate document will contain the master list of only names and study numbers.

Organizations that may inspect and/or copy your research records for quality assurance and data analysis include groups such as: The Faculty of Graduate Studies at the University of Manitoba and The Master of Science in Medical Rehabilitation Department at The School of Medical Rehabilitation of The University of Manitoba.

The University of Manitoba Human Research Ethics Board may review records related to the study for quality assurance purposes.

All records will be kept in a locked secure area and only those persons identified will have access to these records If any of your research records need to be copied to any of the above, your name and all identifying information will be removed. No information revealing any personal information such as your name, address or telephone number will leave The University of Manitoba. 


\section{Voluntary Participation/Withdrawal from the Study}

Your decision to take part in this study is voluntary. You may refuse to participate or you may withdraw from the study at any time. Your decision not to participate or withdraw from the study will not affect your relationship with the other chiropractors in the study. If the study staff feels that it is in your best interest to withdraw you from the study, they will remove you without your consent.

We will tell you about any new information that may affect your willingness to stay in the study.

\section{Questions}

You are free to ask any questions that you may have about your treatment and your rights as a research participant. If any questions come up during or after the study or if you have a research-related injury, contact the study investigators: Geoff Gelley at 2540130 or Brian MacNeil 977-5635

For questions about your rights as a research participant, you may contact The University of Manitoba, Bannatyne Campus Research Ethics Board Office at (204)7893389.

Do not sign this consent form unless you have had a chance to ask questions and have received satisfactory answers to all of your questions. 


\section{Statement of Consent}

I have read this consent form. I have had the opportunity to discuss this research study with Dr. Geoff Gelley and his study staff. I have had my questions answered by them in language I understand. The risks and benefits have been explained to me. I believe that I have not been unduly influenced by any study team member to participate in the research study by any statements or implied statements. Any relationship (such as employer, supervisor or family member) I have with the study team has not affected my decision to participate. I understand that I will be given a copy of this consent form after signing it. I understand that my participation in this study is voluntary and that I may choose to withdraw at any time. I freely agree to participate in this research study.

I understand that information regarding my personal identity will be kept confidential, but that confidentiality is not guaranteed. I authorize the inspection of any of my records that relate to this study by The University of Manitoba Research Ethics Board for quality assurance purposes. 
By signing this consent form, I have not waived any legal rights that I have as a participant in a research study.

I agree to be contacted for future follow-up in relation to this study: Yes No

Participant signature

Date

(Day/month/year)

Participant printed name: 
Appendix B

The onset of the SMT event was defined relative to the acceleration-derived jerk waveform. For this, jerk was calculated from the acceleration data through numerical differentiation. A baseline segment of the jerk data was then used to calculate the standard deviation of the jerk baseline. A threshold equivalent to two standard deviations was then generated and the onset in the acceleration data was taken as the point at which the jerk waveform exceeded the 2 standard deviation threshold. The typical waveform consisted of a triphasic response as seen in figure 18 .

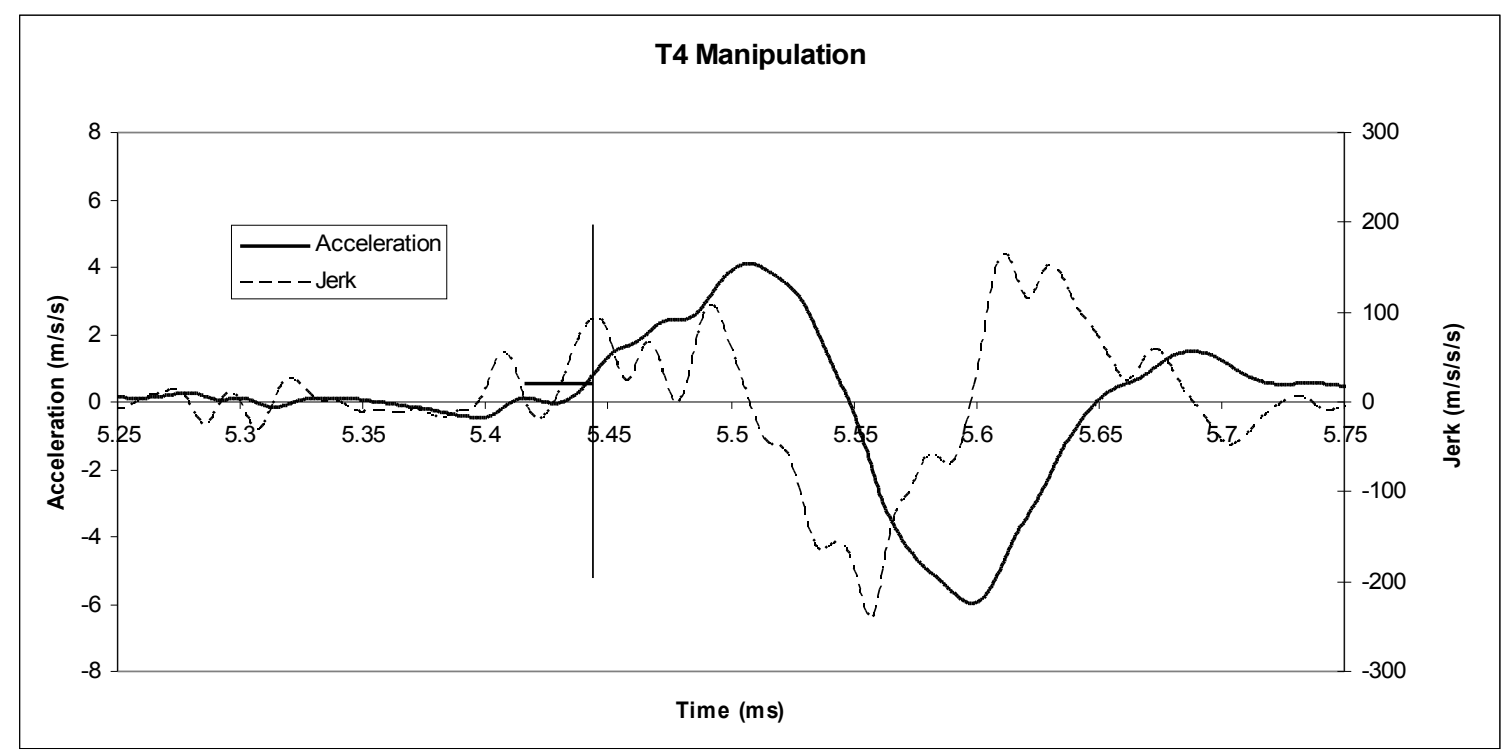

Figure 18. Acceleration and calculated jerk waveform. Vertical line is the peak of the jerk waveform used for the onset of the SMT while the horizontal line represents where the jerk waveform exceeded the 2 standard deviation threshold.

Figure 19 represents a manipulation delivered to the T4 segment of the thoracic spine. From this waveform three distinct phases and the associated peaks can be identified: P1, P2 and P3. For the purposes of this study, only the P1 and P2 phases of the acceleration waveform will be analyzed which corresponds to the intended input of the 
clinician whereas P3 includes unknown contributions from additional factors. The P3 phase appears similar to the resolution phase of the force-time history as outlined by Herzog (2000). He does not consider the resolution phase to be important clinically as it consists of the release of forces following the treatment thrust.

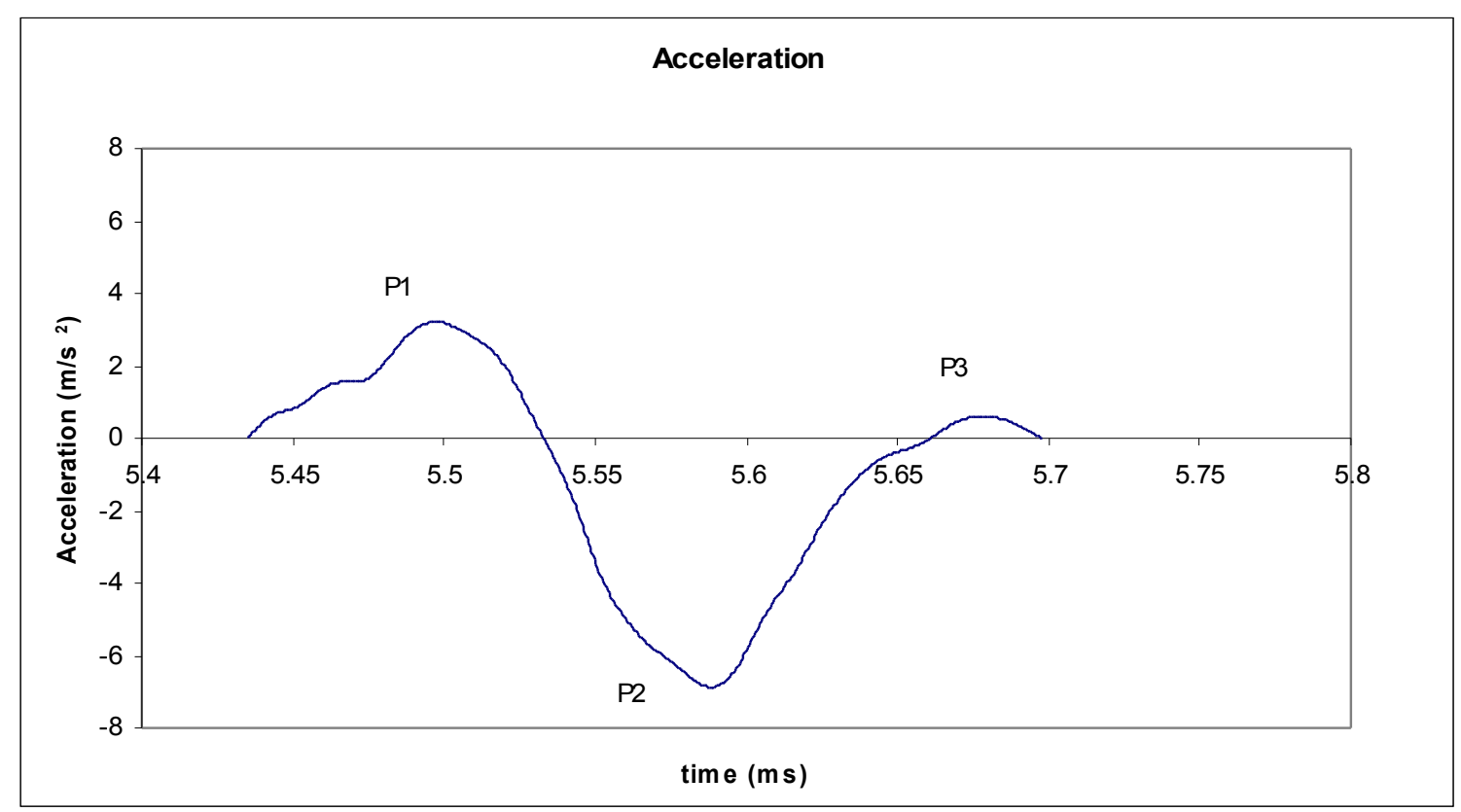

Figure 19. Isolated T4 manipulation with $\mathrm{P} 1, \mathrm{P} 2$ and $\mathrm{P} 3$ labels.

Phase one (P1) represents acceleration of the clinician's hand from the pretensioning position and includes the peak acceleration achieved by the clinician during the SMT event. Phase two (P2) begins when the acceleration signal crosses at the zero point and contains the primary deceleration component including the reversal of the hand following compression and recoil of the tissues. Therefore P2 is likely representative of clinician input and tissue response. P3 is included here for illustrative purposes but the distinct factors that contribute to this phase are uncertain. No portion of P3 will be used for analysis.

From the thoracic acceleration data, velocity and displacement of the clinician's 
hand during the manipulative process was estimated using numerical integration (trapezoid rule) (Figure. 20). Velocity was calculated at all spinal levels, while displacement was not calculated for the cervical, lumbar and sacroiliac regions due to the excessive error that occurs during numerical integration.

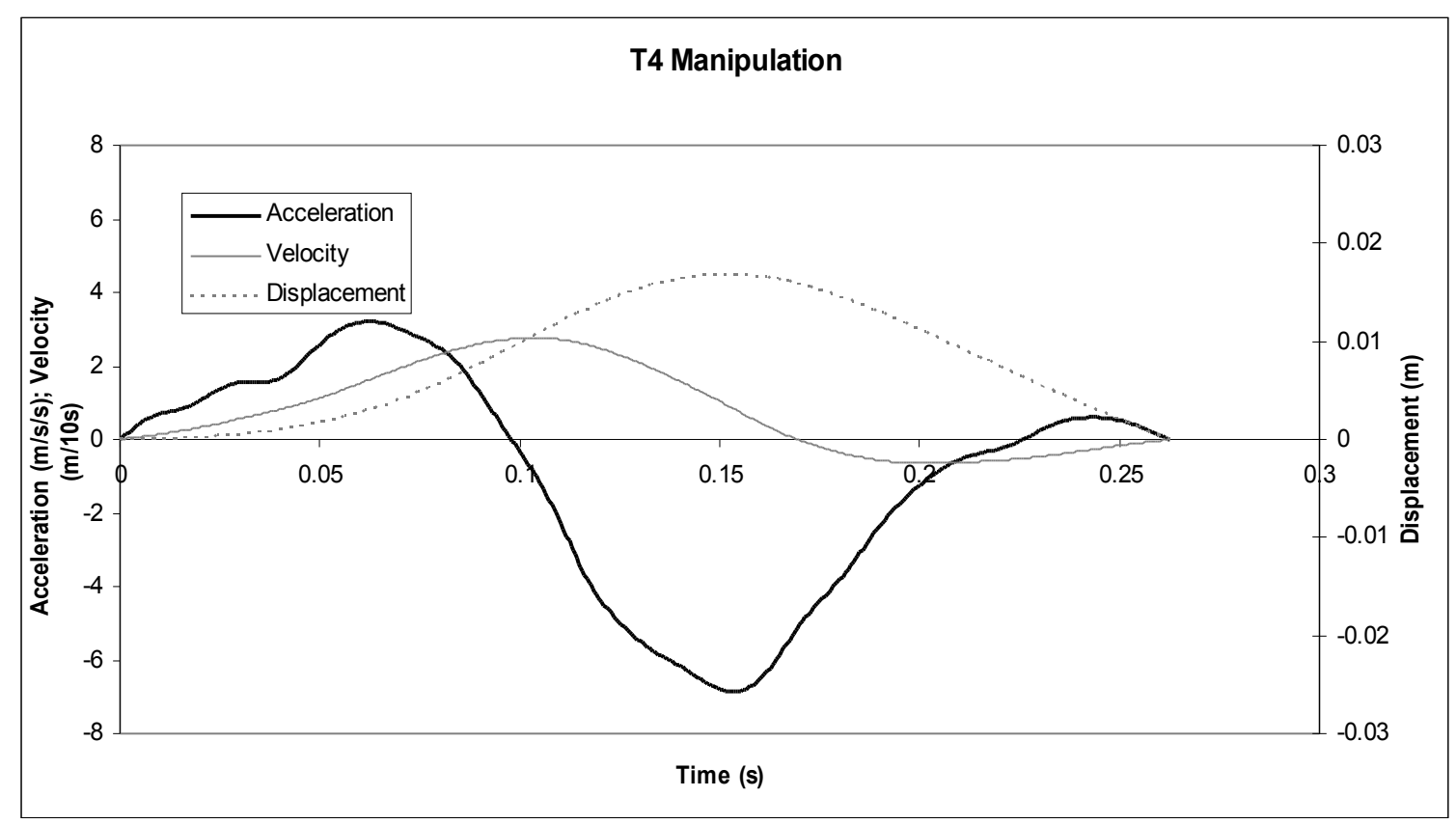

Figure 20. Acceleration and calculated velocity and displacement waveforms.

From each SMT event the following outcome variables will be derived:

1. Jerk: peak amplitude of the waveform which is derived from acceleration utilizing numeric differentiation and was calculated for all of the SMT events except for SI manipulation.

2. Acceleration: peak amplitude and peak latencies for P1, P2 and P1P2. Peak P1 amplitude is the maximum value of the P1 phase while the P1 latency represents the time-based value from the onset of the SMT to peak P1 amplitude. Similarly, peak P2 is the maximum value of the P2 phase 
and P2 latency is the time from the onset of the manipulation to peak P2 amplitude. Finally, peak P1P2 amplitude is the maximum value for the interval from peak P1 to peak P2 amplitude. The time-base value from peak P1 to peak P2 is defined as the P1P2 latency (Figure 21).

3. Other main outcomes included P1 duration (P1_Dura) and P1 area under the curve (P1_AUC) (Figure 21). The P1 duration is the time-base of the P1 phase from the onset of the manipulative event to where the P1 phase returns to zero. P1_AUC is the area under the P1 phase of the acceleration waveform that is calculated using the cumulative trapezoidal rule. The acceleration waveform was also partitioned into the $\mathrm{P} 1$ rise slope $10 \%$ 90\% (P1RS1090), which is calculated by $\mathrm{P} 1$ rise divided by $\mathrm{P} 1$ rise time for $10 \%$ to $90 \%$ of the rise from zero to peak P1. P1RS1090 was determined for all spinal levels except for SI.

4. Velocity (all spinal levels) and displacement (thoracic only): peak magnitudes and latencies. Calculation of velocity and displacement utilizes the numerical integration of acceleration and velocity respectively. The latencies for velocity and displacement are the maximum time-based values from the onset of the manipulation. 


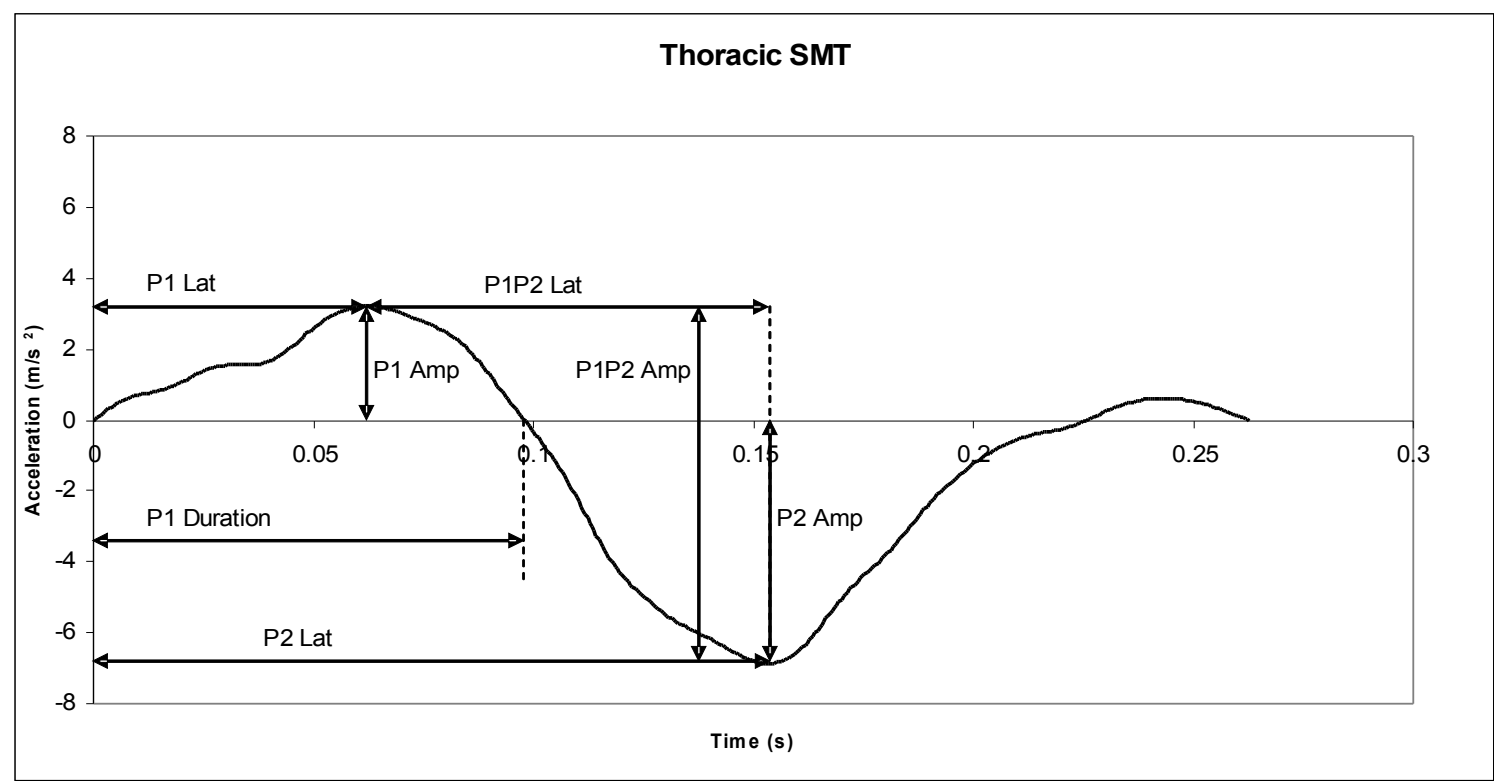

Figure 21. SMT amplitudes and latencies. 